\title{
PEAT DEPOSITS IN IOWA
}

BY

S. W. BEYER 



\section{PEAT DEPOSITS IN』IOWA}

BY S. W. BEYER.

\section{CONTENTS}

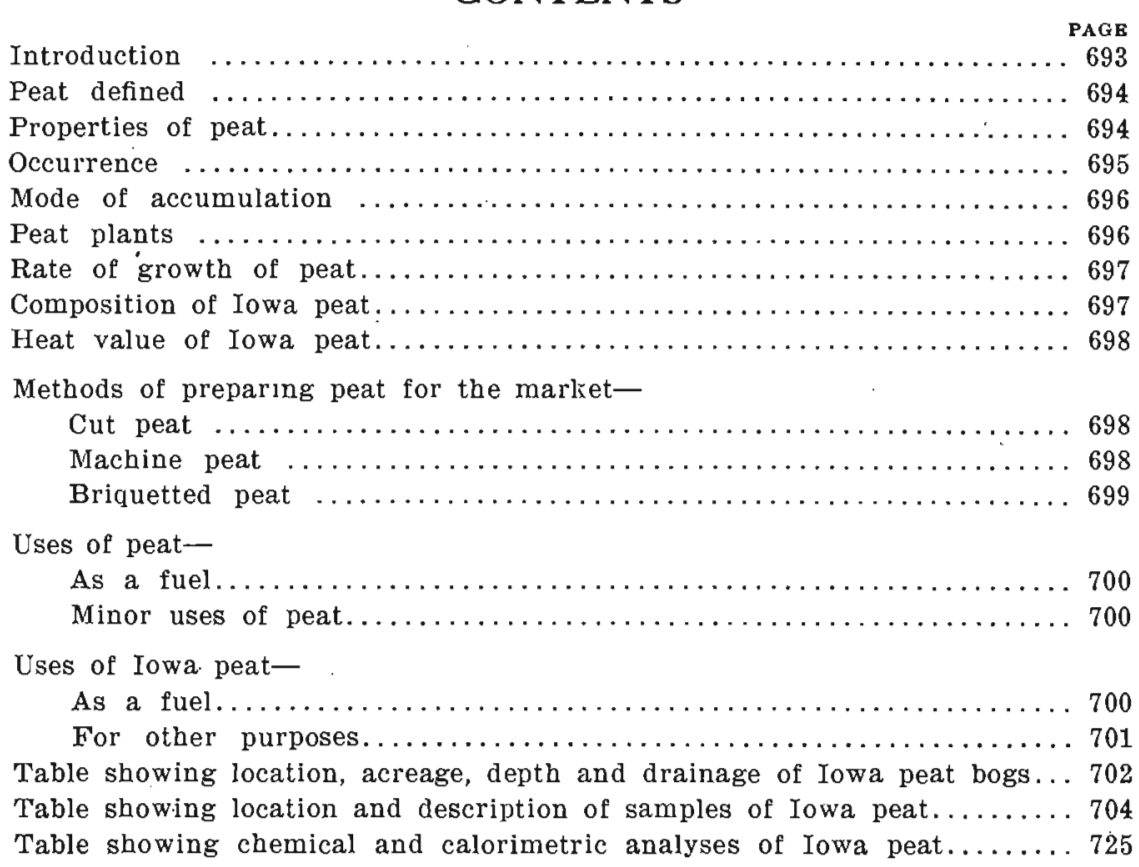





\section{PEAT AND PEAT DEPOSITS IN IOWA}

Introduction. During the summer of 1905 the Iowa Geological Survey detailed Mr. L. H. Wood to locate and determine the area and thickness of the leading peat swamps in Iowa: On the basis of Mr. Wood's notes, Mr. T. E. Savage, then Assistant State Geologist, prepared "A Preliminary Report on the Peat Resources of Iowa" which appeared early in 1906 as Bulletin 2 of the Survey. Several of the county reports published before the appearance of the bulletin casually mentioned the peat deposits within their borders, and all of those published since having to do with counties within the peat area mention and some describe their peat resources.

Several attempts have been made to utilize Iowa peat, notably near Dows in Franklin county and at Goose Lake near Fertile in Worth county. Both plants have not yet passed the experimental stage. Interest in the possibilities of the state's resources in peat has been stimulated greatly by the preliminary work of the Survey, and hence the reason for the present more extended inquiry.

During portions of the summers of 1907 and $1908 \mathrm{Mr}$. W. F. Coover, assisted by Mr. F. A. Knowles during 1908, mapped and sampled the more important bogs in the state. The work of sampling was made difficult because of the protracted wet weather during the field seasons. Bogs containing less than forty acres were not sampled, as a rule, and no samples were saved in those where the vegetable debris averaged less than three feet in thickness. A two-inch common auger was used both for testing depth and securing samples. In general, all of the borings of a hole were mixed and constituted but a single sample. Where considerable changes in character and quality of material were noted an additional sample was sometimes. taken. The samples were numbered and shipped to headquarters. for further study and analysis. 
Peat Defined. Ordinary vegetable matter according to standard authorities is composed of two compounds, cellulose $\left(\mathrm{C}_{6} \mathrm{H}_{10}\right.$ $\mathrm{O}_{5}$ ) or vegetable fiber, and lignin $\left(\mathrm{C}_{35} \mathrm{H}_{24} \mathrm{O}_{20}\right)$ or ordinary wood fiber. Nitrogen in small quantities is generally associated witl the two preceding.

Both cellulose and lignin are unstable under atmospheric conditions and tend to break down to much more simple compounds, chief of which are members of the marsh gas series, carbonic acid gas and water. In air the destruction of vegetable matter. is almost complete, leaving behind only the inorganic residue, similar in character to the ash residue which remains after burning wood. In fact, slow exidation in air under natural conditions produces results practically parallel to those produced by rapid oxidation through burning. In the case of plant remains which accumulate under water, the process of simplification is interfered with and only a partial chemical breaking down is the result. The principal changes which take place are a relative loss in oxygen and liydrogen and an increase in carbon. The resultant partially derayed plant debris is known as peat. From the above it is evident that the physical constitution and chemical composition must be highly variable. Physically peat varies from a highly fibrous, heterogeneous lace work of almost unchanged plant remains to a fiberless, homoguneous, structureless muck or mud. In the first the original plants are easily identified, while in the latter the organic origin can be determined only throngl the assistance of the compound microscope.

In composition the variation is important but less easily marked. The carbon percentage ranges from about forty to over sixty. In color Iowa peat ranges from a light brown through various shades of brown to almost jet black in the nonfibrous varieties. The latter often show shades of gray and blue due to the presence of clay and remains of fresh water shells.

Properties of Peat. Peat when freshly removed from the bog holds from seventy-five to eighty-five or even ninety per cent of water. Even air dried peat retains from ten to twenty-five per cent of water, the amount varying with climatic conditions and the purity and physical constitution of the peat. In general, the freer from impurities and more fibrous the peat, the more water 
retained. It was early observed that animal organisms imbedded in peat were preserved indefinitely. It is due to this antiseptic property that peat itself is preserved from decay.

Occurrence. Most of the peat deposits in Iowa occur in shallow depressions, but occasional peat deposits may be found on gently sloping hillsides, marking a line of seeping springs. The latter are usually of too small extent to be commercially considered. The first type only receives attention in this paper. Various terms have been and are being applied to peat deposits in Iowa. Perhaps the most common are bog, marsh and swamp. The late Professor N. S. Shaler used the term morass in his published writings for similar deposits. Professor C. A. Davis in his report on the Michigan* Peat Deposits uses the above terms for certain specific types of undrained areas. He defines a bog "as an area of wet, porous land on which the soil is made up principally of decayed and decaying vegetable matter, so loosely consolidated, and containing so much water, that the surface shakes and trembles as one walks over it. The vegetation upon the surface is variable, but it is characteristically either some species of moss or of sedge, or grass, or a combination of two or more of these with shrubs and even small trees." A marsh he states "has a firm soil that is not easily shaken when walked upon, although it may be soft and very wet, even submerged, and the vegetation upon it is principally grass-like, that is, with long narrow leaves, and weak, short-lived aerial stems. Slrubs may occur upon marshes, and where they are present not infrequently form thickets." A swamp as described by Davis "has trees and shrubby plants as the most important part of the vegetation, the soil being, as in the case of the marsh, firm, but wet, even, at times, to flooding." It is apparent even to casual observers that there can be no sharp line of separation according to Davis' definitions between the bog, the marsh, and the swamp.

In many cases two or even all of the above types may be represented in the same basin. Practically all of the Iowa peat accumulations worthy of mention belong to the bog type. Swamps as defined by Professor Davis are unknown in Iowa, at least in the peat producing district. All of the peat bogs of

*Annual Report, Geological Survey of Michigan, 1906, pp. 108, et seq. 
commercial importance are confined to the Wisconsin drift sheet, and by far the most important bogs are within the Altamont morainal belt on the east side of the Wisconsin lobe. The bogs vary greatly in area and depth. They range in area up to 1,500 acres and in depth to more than thirty-five feet. The deepest bogs tested are in those morainal tracts where the surface is most broken. Away from the morainal belt the basins become shallower and the peat thinner and as a general rule poorer in quality.

Mode of Accumulation. In many of the shallower basins water plants grow directly from the bottom and peat is accumulated through the successive generations of plants. Most of the bogs in the interior of the Wisconsin drift lobe are of this type. In the deeper bogs the filling is done by the growth of plants from the edges and to some extent from plants which grow on top of the water. The plant border extends itself as a mat over the surface of the water and eventually covers the entire surface of the lake or pond with a floating vegetable mat. Bear Lake in Winnebago county is an example of a lake being slowly transformed into a bog by the extension of the border mat. Sample holes put down at some distance from the open water disclosed a heavy fibrous layer underlain by a very soft peaty mud. The usual sequence in the deeper bogs is a brown, highly fibrous layer or surface mat resting on a non-fibrous to slightly fibrous brownish black mud or muck. In many instances this sequence is repeated, and below the peat mud is a second brown fibrous horizon followed by a second layer of peaty mud. Marly to clayey layers are not infrequently interspersed with the purer peat.

Peat Plants. Professor L. H. Pammel has prepared a list of the leading plants which contribute to the production of Iowa peat deposits. Suffice it to say that while in high latitudes and high altitudes the club mosses, Sphagnums and Hypnums contribute very largely toward the accumulation of peat, grasses and sedges are much more important in lowa peat bogs. Mosses are present occasionally, but play a secondary role. Michigan and Wisconsin bogs support heavy growths of timber in many cases, but lowa bogs are remarkably free from the growth of trees and shrubs. 
Rate of Growth. The rate of accumulation is variable, but must be extremely slow, especially where the smaller plants predominate and there is but little increase through wash. In Iowa, as the chief deposits are in the Wisconsin drift, the filling has probably been going on since the retreat of that ice sheet. The deepest bogs probably exceed thirty-five feet but little. Assuming the minimum time estimate since the disappearance of the Wisconsin ice and the maximum thickness of bog, the maximum rate of accumulation would be about one foot of peat in about two hundred years. The average rate is undoubtedly much less:

Composition of Ioula Peat. Both chemical and calorimetric analyses were made of the peat samples collected. Chemically, Iowa peat runs high in ash and comparatively low in the combustible elements. The leading impurities are quartz sand, and clay, and lime carbonate derived from the remains of small fresh water molluses.

The Goose Lake peat bog near F'ertile in Worth county may be considered fairly representative of the best grade of Iowa peat bogs. An average sample of the air-dried machine peat put upon the market by the Fertile Peat and Clay Company gave the following composition:

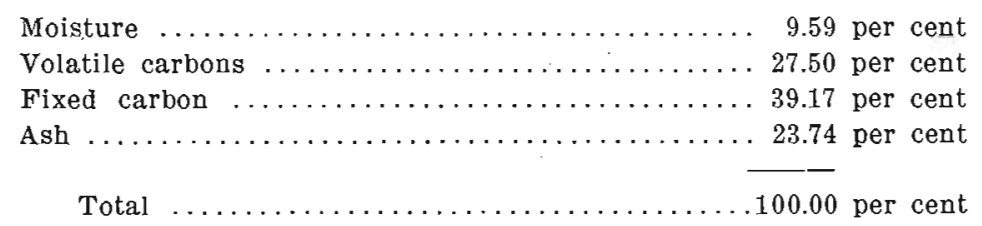

An analysis of ash from the above sample gave the following results :

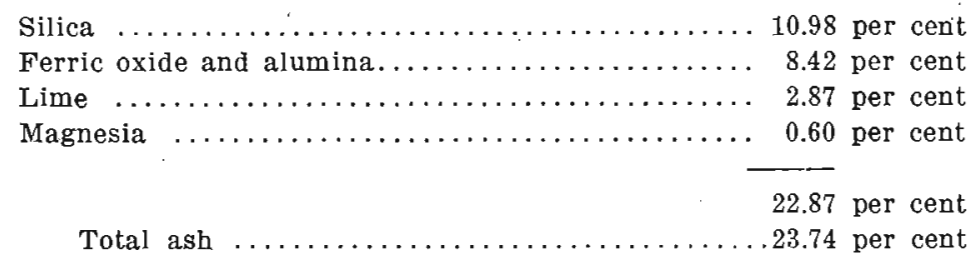

In this particular case the leading impurity is clay with some lime. On account of the prevailingly high percentages of ash, the heat values run correspondingly low. 
The high percentage of ash in Iowa peat as compared with Michigan, Ontario and Maine peat, as well as the increase in ash toward the southwest in the Iowa field, is believed to be due largely to wind blown silt and clay. The prairie character of the Iowa bogs would interfere less with wind work than the timbered areas to the north and east.

The Ileat Value of Iowa Peat. Davis in his work on Michigan peat shows that, in general, the heat value of peat varies inversely as the amount of ash present, but that there are many exceptions to the rule. The same rule applies to Iowa peat. A casual inspection of the analyses appended lerewith is convincing and the dependence of heat value to ash is obvious. The range is from about 8,400 British Thermal Units down to peats so low in combustible matter that the charge fails to explode in the calorimeter. The average Iowa peat has about one-half the heat value of the average Iowa coal.

\section{METHODS OF PREPARING PEAT FOR THE MARKET}

Cut Peat. In Western Europe peat has long been used as a fuel by the common people. The method earliest employed and still used extensively was to cut the peat into rectangular blocks which were ricked up on or near the bog and permitted to air dry. This method involves no treatment whatever, and the product is known as cut peat. Only the fibrous varieties can be used in this way. On account of the bulkiness of the product and loss in handling, the use of cut peat is limited to the immediate vicinity of the bog. The cut peat retains from twenty to forty per cent of moisture.

Machine Peat. The essential features in the production of machine peat are that the peat is treated either in the bog or after its removal from the bog, with or without the addition of water, and then moulded by hand or by machinery. The simplest possible method is where the peat after being ont loose is worked into a pulp by the tramping of men or animals. Then it is moulded into blocks of convenient size and shape and dried in air. Machine peat generally means the use of machinery, and in those plants considered up-to-date, the removal from the pit, the reduction to a peat pulp, and the final moulding into blocks are 
all done by machinery. A considerable number of excavators, conveyors, tempering and moulding machines have been invented and are in use in western and northern Europe. Some of these have been imported, modified, and are in use on the North American continent. The leading foreign types take the names of their inventors, and are the Schlickeysen,* R. Dolberg, A. Heinen, L. Lucht, A. Anrep, Svedala, and Alierman. In all of the above the peat is reduced to a pulp by passing through a machine resembling either the disintegrater or the pug mill, or both, used in preparing clay in ordinary brick plants. The peat plant near Fertile in Worth county las installed a Heinen machine.

In all of the above machines the peat pulp issues as a continuous bar, is received on pallets, is cut into convenient lengtlis and "removed to the drying field or driex". As a rule artificial beat is not used in the drying of machine peat. The air dried machine peat retains from fifteen to thirty per cent of moisture.

Briquetted Peat. In botl cut peat and machine peat the low density and consequent bulliness and low fuel value of the product, its high moisture content and friable character not only render transportation and repeated handling unprofitable. but practically prohibit them. To overcome these difficulties, a number of briquetting machines have been devised and put into use. The fundanental processes in bricuetting consist of first removing a pericn of the water from the peat, after which it is passed through a disintogrator, screened, thoroughily dried in a rotary or plate drier, and then put through the briquette machine. A plunger working in a die subjects each briquette momentarily to a pressure of from 15,000 to 30,000 pounds per square incl.. The resultant product possesses nearly the density of bituminous coal and is but slowly affected by the weathering. agencies.

Attempts have been made to briquette peat direct from the pit, but without success. No mechanical device has yet been found by which the water can be removed directly.

* Peat and Lignite, their Manufacture in Europe; by E. Nystrom, Canada Department of Mines. Mines Branch, pp. $58-81$; Ottawa, 1908 . 


\section{USES OF PEAT}

As a Fuel. The principal use of peat has been and is as a fuel. It may be used direct as cut peat, machine peat, or briquettes, or may be transformed into coke, half coke, producer gas, or powder before burning. The latter two methods deserve most consideration at present for Iowa peat. Producer gas has been used much more extensively in western Europe than in the United States, and especially is this true of producer gas generated from peat. The gas obtained from peat compares very favorably in quality and heat value with that obtained from coal. $\mathrm{Ny}$ strom* reports that peat used as a powder and containing seventeen per cent of water gave nearly the same energy as the same weight of Newcastle coal. Peat as a source of producer gas must be of interest to north central Iowa where other fuel is scarce.

In the production of producer gas from peat, tar and ammonium sulphate may be recovered as by-products.

Minor Uses of Peat. On account of its high absorbent power it is used as stable litter, as a deodorizer and disinfectant, as an antiseptic and absorbent in surgery. It is also used as a fer-' tilizer for lands deficient in humus. Fibrous peat is used as a packing material, for paving and building block, and artificial lumber, and for the manufacture of paper, and even woven into cloth.

\section{USES OF IOWA PEAT}

As a Fuel. From a casual inspection of the table of chemical analyses it is obvious that a great majority of the samples of Iowa peat analyzed carry upwards to twenty-five per cent of ash. According to Davis in his report on Michigan peat bogs, peat carrying more than twenty or twenty-five per cent of ash is too low grade to be considered commercially. Samples carrying up to thirty or thirty-five per cent burn readily and may be considered a serviceable fuel where other fuels are scarce. Such low grade fuel could not be shipped far from the bog in competition with Iowa and Illinois coals. It is possible that ways and means may be found to transform the peat into power through the producer gas engine and transmit it electrically to points where needed, or into a gas and pipe it from the bog to the consumer.

*Peat \& Lignite, Etc. ; Canada Dept. Mines, pp. 171 and 172 , and 198 et seq.; Ottawa,
1908 . 
Davis reports that a ton of peat carrying twenty to twenty-five per cent of moisture treated by the Ziegler process yields 6,650 cubic feet of gas, while the best English cannel coals yield scarcely twice as much gas. The cost per thousand feet is decidedly in favor of the peat. The peat coke is far superior to the gas coke on account of its freedom from sulphur and other objectionable impurities.

The ordinary gas producers now on the market in the United States are not adapted for the use of peat. Several of the large manufacturers of producers are at work on the problem and a producer which will successfully use peat is confidently expected in the near future.

The peat machined at Fertile and Dows kindles readily, burns without clinkers, and gives fair satisfaction for domestic purposes.

For Other Purposes. A sample of peat from the Fertile plant was sent to the Pilgrim Paper Company, Capac, Michigan, for examination, but was pronounced unsuited for the manufacture of paper pulp. The brown, highly fibrous layer found in the majority of Iowa bogs ought to furnish material sufficiently fibrous for a paper pulp, This superficial layer varies from two or three feet up to eight or ten feet in thickness. On account of its high absorptive capacity, it ought to find a ready sale for litter and packing material as straw and excelsior become scarcer. 


\section{TABLE I}

Shows location by county, township, and section; acreage, average depth, and character of drainage of the more important Iowa peat bogs. The last column contains sample numbers representing each bog and corresponding to sample numbers in two tables following.

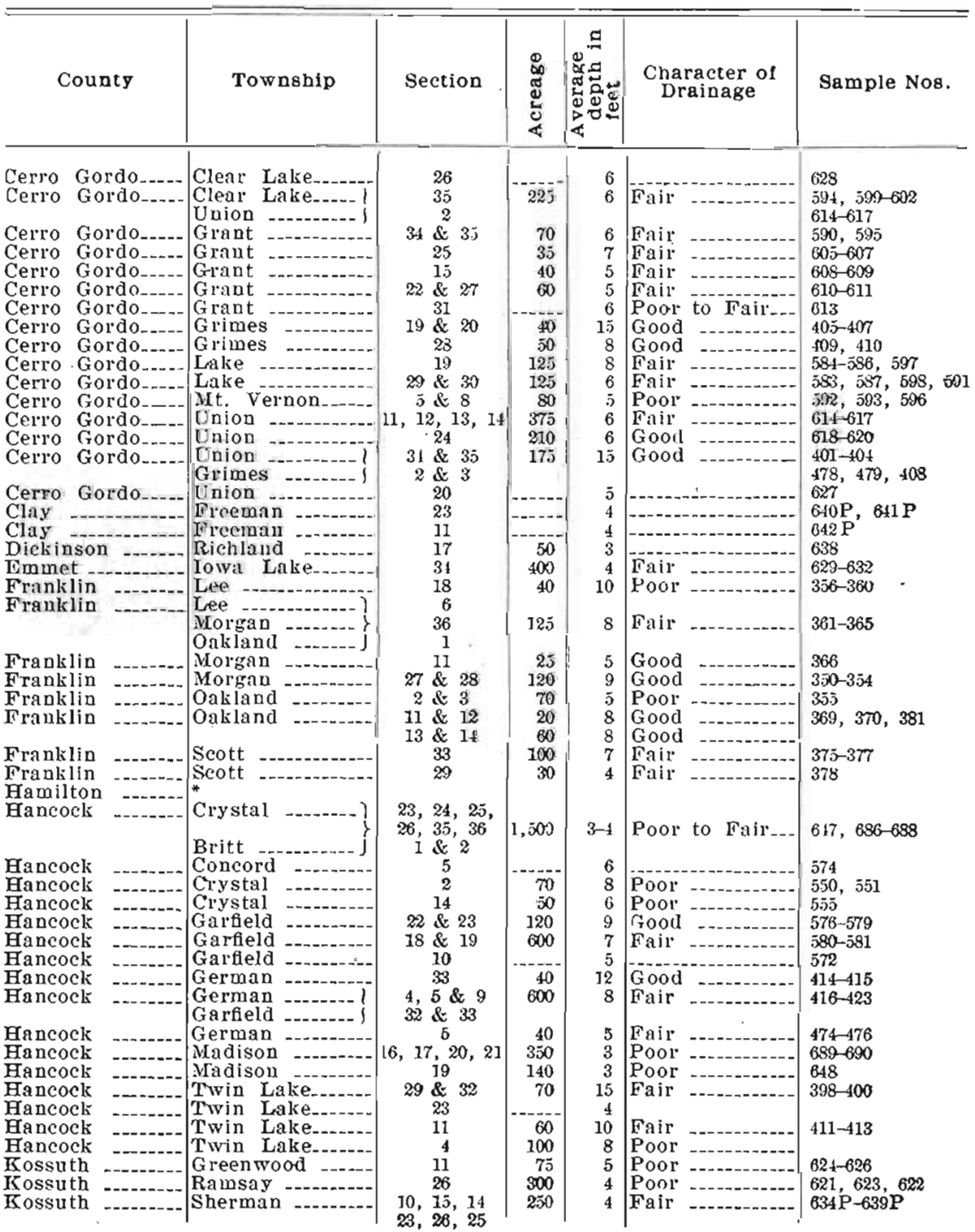

*Several areas or thin peat, Iowa Lake perhaps the most important. 
TABLE I-CONTINOED

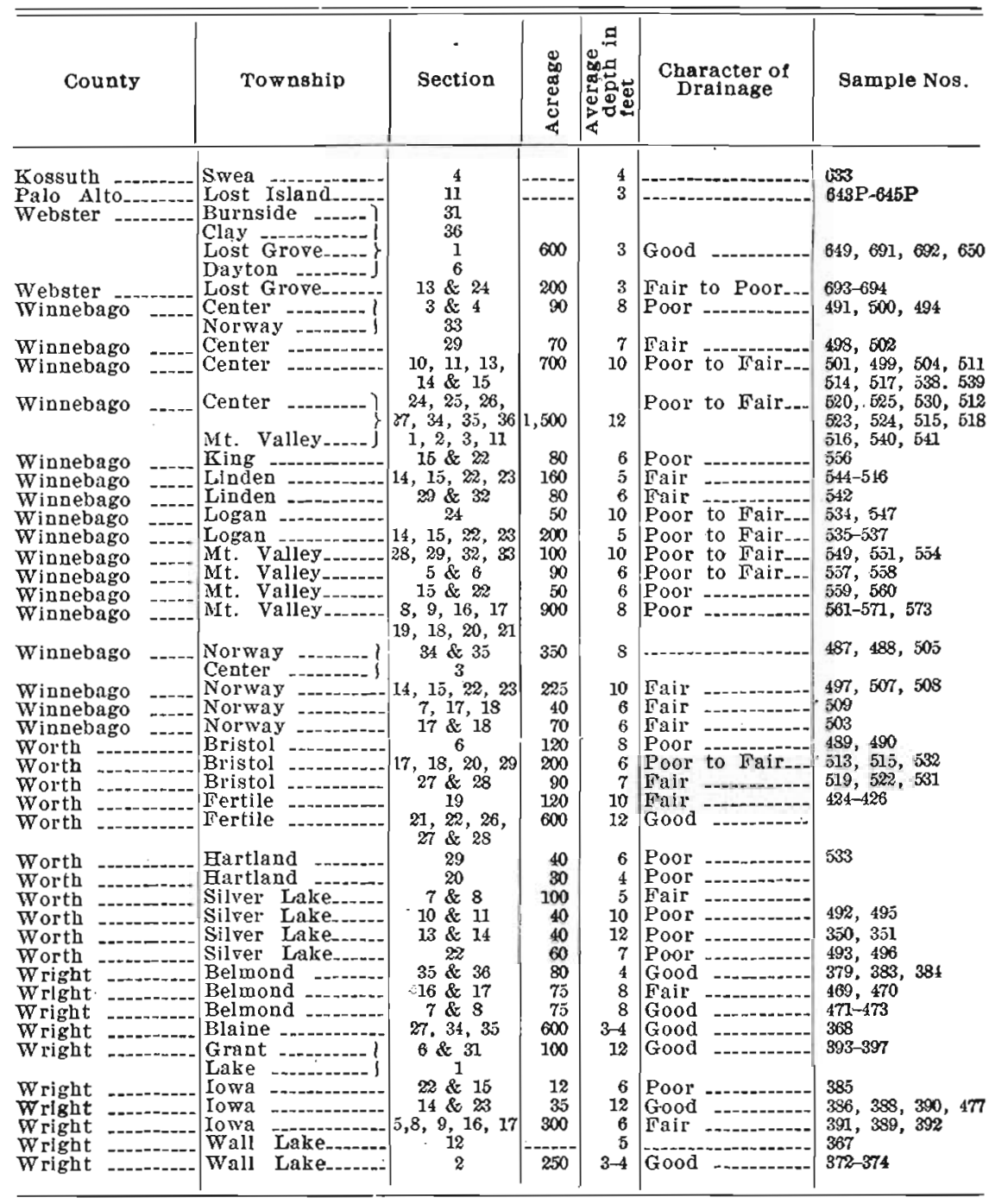


TABLE II

Contains laboratory number, detailed location, and detailed description for each peat sample studied.

\begin{tabular}{|c|c|c|c|}
\hline Lab.No. & County & Detailed Location of Sample & Description of Section \\
\hline 350 & Franklin & $\begin{array}{l}\text { Morgan Twp., Sec, } 28 . \\
\text { Near west end of bog }\end{array}$ & $\begin{array}{l}4 \text { ft. brown, fibrous. } \\
4 \text { ft. brownish black, less } \\
\text { fibrous. } \\
8 \mathrm{ft} \text { sand and peat. }\end{array}$ \\
\hline 351 & Franklin & $\begin{array}{l}\text { Morgan Twp., Sec. } 28 . \\
\text { Near pit of Iowa Peat } \\
\text { Plant }\end{array}$ & $\begin{array}{l}3.5 \text { ft. brown, fibrous. } \\
4 \text { ft. brown and black } \\
\text { mingled. }\end{array}$ \\
\hline 352 & Franlslin & $\begin{array}{l}\text { Morgan Twp., Sec. } 28 . \\
200 \text { yds. Se. sample No. } \\
351 .\end{array}$ & $\begin{array}{l}5.5 \text { ft. brown, fibrous. } \\
5 \text { ft. brownish black, but } \\
\text { slightly fbrous. }\end{array}$ \\
\hline 353 & Franklin & $\begin{array}{l}\text { Morgan Twp., Sec. } 28 . \\
200 \text { yds. S. of east of } \\
\text { sample. No. } 352\end{array}$ & $\begin{array}{l}6.5 \mathrm{ft} \text {. brown, fibrous. } \\
2 \mathrm{ft} \text {. brownish black, non- } \\
\text { fibrous. } \\
1.5 \mathrm{ft} \text { brown, fibrous }\end{array}$ \\
\hline 354 & Franklin & $\begin{array}{l}\text { Morgan Twp., Sec. } 27 . \\
320 \text { yds. S. of east of } \\
\text { sample No. } 353\end{array}$ & $\begin{array}{l}8 \text { ft. brown, fibrous. } \\
2 \text { ft. brownish black, non- } \\
\text { fibrous. } \\
\text { Blue clay below. }\end{array}$ \\
\hline 355 & Franklin & $\begin{array}{c}\text { Oakland Twp., Sec. } 3.300 \\
\text { yds. from Ne. corner }\end{array}$ & $\begin{array}{l}6.5 \mathrm{ft} \text {. brown, fibrous. } \\
\text { A thin layer of non-fibrous } \\
\text { below. }\end{array}$ \\
\hline 356 & Franklin & $\begin{array}{l}\text { Lee Twp., Sec. } 18 . \text { On } \\
\text { north line }\end{array}$ & $\begin{array}{l}2.5 \mathrm{ft} \text {. brown, fibrous. } \\
3 \mathrm{ft.} \text { brownish black, non- } \\
\text { fibrous. }\end{array}$ \\
\hline 357 & Franklin & $\begin{array}{l}\text { Lee Twp., Sec. } 18 \text {. On } 1 / 2 \\
\text { Sec. line, } 125 \text { yds. from } \\
\text { edge of bog }\end{array}$ & $\begin{array}{l}8 \text { ft. brown, fibrous. } \\
2 \text { ft. brownish black, non- } \\
\text { fibrous. } \\
5 \text { ft. brown, fibrous. }\end{array}$ \\
\hline 358 & Franklin & $\begin{array}{l}\text { Oakland Twp., Sec. } 11 . \\
\text { On Sec. line (Secs. } 11 \\
\text { and 12) }\end{array}$ & $\begin{array}{l}8 \text { ft. brown, fibrous. } \\
9 \text { ft. brownish black, non- } \\
\text { fibrous. } \\
\text { Thin clay seam at } 14 \mathrm{ft} .\end{array}$ \\
\hline 359 & Franklin & $\begin{array}{l}\text { Oakland Twp., Sec. } 11 . \\
\text { Near south end of bog }\end{array}$ & $\begin{array}{l}7 \mathrm{ft} \text {. brown, fibrous. } \\
7.5 \mathrm{ft} \text {. brownish black, non- } \\
\text { fibrous. } \\
\text { Gravel below. }\end{array}$ \\
\hline 360 & Franklin & $\begin{array}{c}\text { Oakland Twp., Sec. } 12 . \\
\text { East of sample No. } 359\end{array}$ & $\begin{array}{l}7 \text { ft. brown, fibrous. } \\
7.5 \text { ft. brownish black, non- } \\
\text { fibrous. }\end{array}$ \\
\hline
\end{tabular}


TABLE II-CONTINUED

\begin{tabular}{|c|c|c|c|}
\hline LaZ. No. & County & Detailed Location of Sample & \multirow{2}{*}{$\begin{array}{l}\text { Description of Section } \\
\text { 7.5 ft. brown, fibrous. } \\
3.5 \text { ft. brownish black, non- } \\
\text { fibrous. }\end{array}$} \\
\hline 361 & Franklin & $\begin{array}{l}\text { Oakland Twp., Sec. } 1.400 \\
\text { yds. E. 1/2 Sec. line }\end{array}$ & \\
\hline 362 & Franklin & $\begin{array}{l}\text { Oakland Twp., Sec. } 1 . \\
\text { South side of bog }\end{array}$ & $\begin{array}{l}7 \text { ft. brown, fibrous. } \\
4.5 \text { ft. brown ish black, non- } \\
\text { fibrous. }\end{array}$ \\
\hline 363 & Franklin & $\begin{array}{l}\text { Oakland Twp., Sec. } 1 . \\
\text { Near east line section }\end{array}$ & $\begin{array}{l}7 \text { ft. brown, fibrous. } \\
8 \text { ft. brownish black, non- } \\
\text { fibrous. } \\
5.5 \text { ft. brown, fibrous. }\end{array}$ \\
\hline 364 & Franklin & $\begin{array}{l}\text { Morgan Twp., Sec. } 36 \text {, Se. } \\
1 / 4.125 \text { yds. from east } \\
\text { edge of bog }\end{array}$ & $\begin{array}{l}11 \mathrm{ft} \text { brown, fibrous. } \\
1 \mathrm{ft} \text {. brownish black, non- } \\
\text { fibrous. } \\
\text { Clay parting at } 8 \mathrm{ft} \text {. }\end{array}$ \\
\hline 365 & Franklin & $\begin{array}{l}\text { Morgan Twp., Sec. } 36.125 \\
\text { yds. W. of sample No. } \\
364\end{array}$ & $\begin{array}{l}11 \mathrm{ft} \text {. brown, fibrous. } \\
2.5 \mathrm{ft} \text { brownish black. }\end{array}$ \\
\hline 366 & Franklin & $\begin{array}{l}\text { Morgan Twp., Sec. } 11 . \text { On } \\
\text { road } 500 \text { yds. south of } \\
\text { corner }\end{array}$ & $\begin{array}{l}2.5 \mathrm{ft.} \text { brown, fibrous. } \\
3.5 \mathrm{ft.} \text { brownish black. }\end{array}$ \\
\hline 367 & Wright & $\begin{array}{l}\text { Wall Lake Twp., Sec. } 12 . \\
3 / 8 \text { mi. S. north line, } \\
\text { along ditch }\end{array}$ & $\begin{array}{l}3 \mathrm{ft} \text {. brown, fibrous. } \\
3.5 \mathrm{ft} \text {. brownish black. }\end{array}$ \\
\hline 368 & Wright & $\begin{array}{l}\text { Blaine Twp., Sec. } 27 . \text { Near } \\
\text { Nw. corner Sw. quarter }\end{array}$ & $\begin{array}{l}2.5 \mathrm{ft} \text {. brown, fibrous. } \\
4 \mathrm{ft} . \text { brownish black. }\end{array}$ \\
\hline 369 & Franklin & $\begin{array}{l}\text { Oakland Twp., Sec. } 11 . \\
\text { Southeast corner }\end{array}$ & $\begin{array}{l}7 \mathrm{ft} \text {. brown, fibrous. } \\
3.5 \mathrm{ft} \text {. brownish black. } \\
3.5 \mathrm{ft} \text { brown, fibrous. }\end{array}$ \\
\hline 370 & Franklin & $\begin{array}{l}\text { Oakland Twp., Sec. } 11 . \\
300 \text { yds. W. of north of } \\
\text { sample No. } 369\end{array}$ & $\begin{array}{l}7 \text { ft. brown, fibrous. } \\
3 \text { ft. brownish black. }\end{array}$ \\
\hline$\overline{371}$ & Franklin & $\begin{array}{l}\text { Oakland Twp., Sec. } 11 . \\
125 \text { yds. west of sample } \\
\text { No. } 370\end{array}$ & $\begin{array}{l}8 \mathrm{ft} \text {. blownish black. } \\
5.5 \mathrm{ft} \text { lighter colored peat. }\end{array}$ \\
\hline 372 & Wright & $\begin{array}{l}\text { Blaine Twp., Sec. } 35 . \\
\text { yds. south of middle } \mathrm{N} \text {. } \\
\text { line }\end{array}$ & $\begin{array}{l}2.5 \mathrm{ft} . \text { brown, fibrous. } \\
3.5 \mathrm{ft} \text {. brownish black. }\end{array}$ \\
\hline 373 & Wright & $\begin{array}{c}\text { Blaine Twp., Sec. } 35 . \text { Near } \\
\text { center Nw. } 1 / 4 \text { of Ne. } 1 / 4\end{array}$ & $\begin{array}{l}2 \text { ft. brown, fibrous. } \\
3 \text { ft. brownish black. }\end{array}$ \\
\hline 374 & Wright & $\begin{array}{l}\text { Blaine Twp., Sec. } 35 . \quad 140 \\
\text { yds. Sw. sample No. } 373, \\
\text { along fence }\end{array}$ & $\begin{array}{l}2 \text { ft. brown, fibrous. } \\
1.5 \text { ft. brownish black. }\end{array}$ \\
\hline
\end{tabular}


PEAT DEPOSITS IN IOWA

TABLE II-CONTINUED

\begin{tabular}{|c|c|c|c|}
\hline Lab.No. & County & Detailed Location of Sample & Description of Section \\
\hline 375 & Franklin & $\begin{array}{l}\text { Scott Twp., Sec. } 33 \text {. North } \\
\text { section line roac }\end{array}$ & $\begin{array}{l}2 \text { ft. brown, fibrous. } \\
5.5 \text { ft. brownish black. }\end{array}$ \\
\hline 376 & Franklin & $\begin{array}{l}\text { Scott Tru., Sec. } 33 . \\
\text { yds. from middle east } \\
\text { edge of bog }\end{array}$ & $\begin{array}{l}3 \text { ft. brown, fibrous. } \\
3 \text { ft. brownish black. } \\
1 \text { ft. bluish peaty mud. }\end{array}$ \\
\hline 377 & Franklin & $\begin{array}{l}\text { Scott Twp., Sec. } 33 \text {. Near } \\
\text { middle south side of bog }\end{array}$ & $\begin{array}{l}2 \text { ft. brown, fibrous. } \\
3 \text { ft. brownish black. } \\
1 \text { ft. marly layer. } \\
2 \text { tt. brown, fbrous. }\end{array}$ \\
\hline 378 & Franklin & $\begin{array}{l}\text { Scott Twp., Sec. } 29 \text {. Near } \\
\text { Nw. corner at bridge }\end{array}$ & 3.5 ft. brown, fibrous. \\
\hline 379 & Wright & $\begin{array}{l}\text { Belmond Twp. Sec. } 35 . \\
100 \text { yds. north of road } \\
\text { on south line }\end{array}$ & 3 ft. brown, fibrous. \\
\hline 380 & Wright & $\begin{array}{l}\text { Belmond Twp., Sec. } 35 . \\
400 \text { yds. north of road, } \\
\text { east side bog }\end{array}$ & $\begin{array}{l}2 \text { ft. brown, fibrous. } \\
2 \text { ft. peat and grit. }\end{array}$ \\
\hline 381 & Wright & $\begin{array}{l}\text { Belmond Twp., Sec. } 35 . \\
100 \text { yds. directly west of } \\
\text { sample No. } 380\end{array}$ & 4 ft. blownish black peat. \\
\hline 382 & Wright & $\begin{array}{l}\text { Belmond Twp., Sec. } 35 . \\
150 \text { yds. Ne. from sam- } \\
\text { ple No. } 381\end{array}$ & $4.5 \mathrm{ft}$. brownish black. \\
\hline 383 & Wright & $\begin{array}{l}\text { Belmond Twp., Sec. } 36 . \\
\text { Ne. corner Ne. } 1 / 4 \text { of Sw. } \\
1 / 4\end{array}$ & $\begin{array}{l}2 \mathrm{ft} \text {. brown, fibrous. } \\
2.5 \mathrm{ft} \text {. brownish black. }\end{array}$ \\
\hline 383.5 & Wright & $\begin{array}{l}\text { Belmond Twp., Sec. } 35 . \\
\text { West of sample No. } 379\end{array}$ & $\begin{array}{l}2 \text { ft. brown, fibrous. } \\
3 \text { ft. brownish black. }\end{array}$ \\
\hline 384 & Wright & $\begin{array}{l}\text { Belmond Twp., Sec. } 35 . \\
\text { On road south end of } \\
\text { bog }\end{array}$ & $\begin{array}{l}2 \mathrm{ft} \text {. brown, fibrous. } \\
2.5 \mathrm{ft.} \text { brownish black. }\end{array}$ \\
\hline 385 & Wright & $\begin{array}{l}\text { Iowa Twp. line, Secs. } 15 \\
\text { and } 22 \text {. About } 200 \text { yds. } \\
\text { E. of } S w \text {. corner }\end{array}$ & $\begin{array}{l}5 \text { ft. brown, fibrous. } \\
2 \text { ft. brownish black. } \\
1 \text { ft. peaty clay. } \\
2 \text { ft. brownish black. } \\
1 \text { ft. brown, fbrous. }\end{array}$ \\
\hline 386 & Wright & $\begin{array}{l}\text { Iowa. Twp., Sec. } 23 . \text { Near } \\
\text { Nw. corner Ne. } 1 / 4 \text { of } \\
\text { Nw. } 1 / 4\end{array}$ & $\begin{array}{l}5 \text { ft. dark brown. } \\
4.5 \text { ft. marly peat, on gravel. }\end{array}$ \\
\hline
\end{tabular}


TABLE II-CONTINUED

\begin{tabular}{|c|c|c|c|}
\hline Lab.No. & County & Detailed Location of Sample & Description of Section \\
\hline 387 & Wright & $\begin{array}{l}\text { Iowa Twp., Sec. } 23 . \\
\text { yds. south of sample No. } \\
386\end{array}$ & $\begin{array}{l}5 \mathrm{ft} \text {. brownish black, almost } \\
\text { non-fibrous. } \\
1.5 \mathrm{ft} \text {. peaty marl. } \\
13.5 \mathrm{ft} \text {. brown, fibrous. }\end{array}$ \\
\hline 388 & Wright & $\begin{array}{l}\text { Iowa Twp., Sec. } 23.175 \\
\text { yds. due east sample No. } \\
387 \text {. }\end{array}$ & $\begin{array}{l}2.5 \text { ft. brownish black. } \\
9.5 \text { ft. peaty marl. }\end{array}$ \\
\hline 389 & Wright & $\begin{array}{c}\text { Iowa Twp., between Secs. } \\
9 \text { and } 16 . \text { At bridge }\end{array}$ & $\begin{array}{l}5.5 \mathrm{ft} \text {. brown, fibrous. } \\
3 \mathrm{ft} \text {. peaty clay. }\end{array}$ \\
\hline 390 & Wright & $\begin{array}{l}\text { Iowa Twp., Sec. } 23 \text {. Near } \\
\text { middle north side of } \\
\text { Nw. } 1 / 4 .\end{array}$ & $\begin{array}{l}2.5 \mathrm{ft} \text {. brownish black. } \\
11 \text { ft. peaty grit and clay } \\
\text { to clayey peat. }\end{array}$ \\
\hline 391 & Wright & $\begin{array}{l}\text { Iowa Twp., between Secs. } \\
8 \text { and } 17 \text {. Near west } \\
\text { edge of bog }\end{array}$ & $\begin{array}{l}2 \text { ft. brown, fibrous, to } \\
\text { black peat mud. }\end{array}$ \\
\hline 392 & Wright & $\begin{array}{l}\text { Iowa Twp., between Secs. } \\
9 . \text { and } 16.60 \text { yds. east } \\
\text { of sample No. } 389\end{array}$ & $\begin{array}{l}5.5 \mathrm{ft} \text {. brown, fibrous. } \\
3 \mathrm{ft} \text {. peaty clay. }\end{array}$ \\
\hline 393 & Wright & $\begin{array}{l}\text { Lake Twp., on E. line Sec. } \\
\text { 1. Lake at bridge near } \\
\text { south edge of bog }\end{array}$ & $\begin{array}{l}3.5 \text { ft. brown, fibrous. } \\
9.5 \text { ft. brownish black. } \\
\text { Sand and gravel below. }\end{array}$ \\
\hline 394 & Wright & $\begin{array}{l}\text { Lake Twp., Sec. 1. About } \\
\text { middle of bog }\end{array}$ & $\begin{array}{l}5.5 \text { ft. brown, fibrous. } \\
14 \text { ft. brownish black. } \\
\text { Grades downward into } \\
\text { black peaty mud. }\end{array}$ \\
\hline 395 & Wright & $\begin{array}{l}\text { Lake Twp., Sec. } 1 . \text { Near } \\
\text { north edge of bog }\end{array}$ & $\begin{array}{l}4.5 \mathrm{ft} \text {. brown, fibrous. } \\
13.5 \text { ft. brownish black, } \\
\text { very sticky below. }\end{array}$ \\
\hline 396 & Wright & $\begin{array}{l}\text { Lake Twp., Sec. } 1 \text {. Along } \\
\text { dredge ditch near north } \\
\text { edge of bog }\end{array}$ & $\begin{array}{l}4.5 \mathrm{ft} \text {. brown, fibrous. } \\
7 \mathrm{ft} \text {. brown ish black. } \\
\text { Gravel and sand below. }\end{array}$ \\
\hline 397 & Wright & $\begin{array}{l}\text { Lake Twp., Sec. 1. Along } \\
\text { ditch } 150 \text { yds. south of } \\
\text { sample No. } 396\end{array}$ & $\begin{array}{l}6 \mathrm{ft} . \text { brown, fibrous. } \\
18 \mathrm{ft} \text {. brownish black. } \\
\text { Bottom not reached. }\end{array}$ \\
\hline 398 & Hancock & $\begin{array}{l}\text { Twin Lakes Twp., Sec. } 29 . \\
\text { Near south edge, } 300 \\
\text { yds. from E. end of bog }\end{array}$ & $\begin{array}{l}4 \mathrm{ft} \text {. brown, fibrous. } \\
6.5 \mathrm{ft} \text {. brownish black. } \\
2.5 \mathrm{ft} \text {. brown, fibrous. } \\
3.5 \mathrm{ft} \text { peaty marl and clay. }\end{array}$ \\
\hline 399 & Hancock & $\begin{array}{l}\text { Twin Lakes Twp., Sec. } 29 . \\
\text { Due north sample No. } \\
398 \text {, near edge }\end{array}$ & $\begin{array}{l}2 \mathrm{ft} \text {. brown, fibrous. } \\
22 \mathrm{ft} \text {. brownish black, va- } \\
\text { riable. } \\
\text { Bottom not reached. }\end{array}$ \\
\hline
\end{tabular}


TABLE II-ContinUED

\begin{tabular}{|c|c|c|c|}
\hline Lab. No. & County & Detailed Location of Sample & Description of Section \\
\hline 400 & Hancock & $\begin{array}{l}\text { Twin Lakes Twp., line } \\
\text { Secs. } 29 \text { and } 32 . \\
\text { east edge of bog }\end{array}$ & $\begin{array}{l}5 \mathrm{ft} \text {. brown, fibrous. } \\
2.5 \mathrm{ft} \text {. brownish black. } \\
11.5 \mathrm{ft} \text {. reddish brown. }\end{array}$ \\
\hline 401 & Cerro Gordo & $\begin{array}{l}\text { Grimes Twp., Sec. } 2.150 \\
\text { yds. from west line of } \\
\text { section }\end{array}$ & $\begin{array}{l}5 \text { ft. brown, fibrous. } \\
6 \text { ft. brownish black. } \\
2 \text { ft. reddish brown. }\end{array}$ \\
\hline 402 & Cerro Gordo & $\begin{array}{rrr}\text { Grimes Twp., Sec. } 2 . & 75 \\
\text { yds. N. of sample } & \text { No. } \\
401 \text { and at center of bog }\end{array}$ & $\begin{array}{l}7 \mathrm{ft} \text {. brown, fibrous. } \\
4 \mathrm{ft} \text {. brown, non-fibrous. } \\
6 \text { ft. lighter brown. }\end{array}$ \\
\hline 403 & Cerro Gordo & $\begin{array}{r}\text { Grimes Twp., Sec. } 3 . \text { Near } \\
\text { east edge bog on Nw. } 1 / 4\end{array}$ & $\begin{array}{l}5 \text { ft. brown, fibrous. } \\
4 \text { ft. brown, less fibrous. } \\
7 \text { ft. lighter brown. }\end{array}$ \\
\hline 404 & Cerro Gordo & $\begin{array}{l}\text { Grimes Twp., Sec. } 3 . \\
\text { Along drain at widdle } \\
\text { of bog. North of sam- } \\
\text { ple No. } 403\end{array}$ & $\begin{array}{l}5 \text { ft. brown, fibrous. } \\
5 \text { ft. brown, non-fibrous. } \\
12 \text { ft. lighter brown. }\end{array}$ \\
\hline 478 & Cerro Gordo & $\begin{array}{l}\text { Grimes Twp., Sec. 3. North } \\
\text { of sample No. } 404 \text { near } \\
\text { north edge }\end{array}$ & $\begin{array}{l}3.5 \mathrm{ft} \text {. brown, fibrous. } \\
8 \mathrm{ft} \text {. brownish black, becom- } \\
\text { ing clayey below. } \\
13 \mathrm{ft.} \text { light brown. }\end{array}$ \\
\hline 405 & Cerro Gordo & $\begin{array}{l}\text { Grimes Twp., line Secs. } \\
19 \text { and } 20 . \text { Bridge near } \\
\text { S. edge of bog }\end{array}$ & $\begin{array}{l}9 \mathrm{ft} \text {. brown, fibrous, becom- } \\
\text { ing marly below. } \\
8 \mathrm{ft} \text {. light brown. }\end{array}$ \\
\hline 406 & Cerro Gordo & $\begin{array}{l}\text { Grimes Twp., north of } \\
\text { sample No. } 405 \text { near } \\
\text { middle of bog }\end{array}$ & $\begin{array}{l}3 \text { ft. brown, fibrous, grading } \\
\text { into } 7 \mathrm{ft} \text {. brownish black, } \\
\text { marly below. } \\
13 \text { ft. light brown to gray- } \\
\text { ish peat. }\end{array}$ \\
\hline 408 & Cerro Gordo & $\begin{array}{c}\text { Union Twp., Sec. } 34 . \text { Sw. } \\
\text { 1/4, near Ne. corner }\end{array}$ & $\begin{array}{l}5.5 \mathrm{ft} \text {. brown, fibrous. } \\
4 \mathrm{ft} \text {. brown, non-fibrous. } \\
1.5 \mathrm{ft} \text {. reddish brown. } \\
8 \mathrm{ft} \text {. grayish brown, clayey. }\end{array}$ \\
\hline 409 & Cerro Gordo & $\begin{array}{l}\text { Grimes Twp., Sec. 28. Se. } \\
1 / 4 \text { of Nw. } 1 / 4\end{array}$ & $\begin{array}{l}3 \text { ft. brown, fibrous. } \\
1 \text { ft. brownish black. } \\
\text { Gravel and sand below. }\end{array}$ \\
\hline \multirow[t]{2}{*}{410} & Cerro Gordo & $\begin{array}{l}\text { Grimes Twp., Sec. 28. Se. } \\
1 / 4 \text { of Nw. } 1 / 4\end{array}$ & $\begin{array}{l}3 \text { ft. brown, fibrous. } \\
2 \text { ft. brownish black. }\end{array}$ \\
\hline & Cerro Gordo & $\begin{array}{l}\text { Grimes Twp., Sec. 28. Due } \\
\quad \text { east of sample No. } 410\end{array}$ & $\begin{array}{l}5 \text { ft. brown, fibrous. } \\
5.5 \mathrm{ft} \text {. brownish black. } \\
5.5 \mathrm{ft} \text {. reddish brown. } \\
7.5 \text { ft. grayish brown, marly. }\end{array}$ \\
\hline
\end{tabular}


TABLE II-CONTINUED

\begin{tabular}{|c|c|c|c|}
\hline Lab. No. & County & Detailed Location of Sample & Description of Section \\
\hline 411 & Hancock & $\begin{array}{l}\text { Twin Lakes Twp., Sec. } 11 . \\
\text { Sw. } 1 / 4 \text { south, middle of } \\
\text { bog }\end{array}$ & $\begin{array}{l}4 \text { ft. brown, coarsely fibrous } \\
\text { Gravel below. }\end{array}$ \\
\hline 412 & Hancock & $\begin{array}{l}\text { Twin Lakes Twp., Sec. } 11 . \\
\text { Sw. } 1 / 4 \text { near center of } \\
\text { bog }\end{array}$ & $\begin{array}{l}5 \text { ft. brown, coarsely fibrous. } \\
8 \text { ft. brownish black. } \\
2 \text { ft. grayish brown. } \\
6 \text { ft. reddish brown. } \\
\text { Sand below. }\end{array}$ \\
\hline 413 & Hancock & $\begin{array}{l}\text { Twin Lakes Twp., Sec. } 11 . \\
\text { Sw. 1/4. North of mid- } \\
\text { dle }\end{array}$ & $\begin{array}{l}5 \text { ft. brown, coarsely fibrous. } \\
5 \text { ft. brownish black. } \\
.5 \text { ft. grayish to reddish } \\
\text { brown. }\end{array}$ \\
\hline 414 & Hancock & $\begin{array}{l}\text { Gelman Twp., Sec. } 33 . \text { E. } \\
1 / 2 \text { of Sw. 1/4. Near east } \\
\text { edge of bog }\end{array}$ & $\begin{array}{l}2 \text { ft. brown, coarsely fibrous. } \\
7 \text { ft. brownish black. } \\
\text { Clayey peat below. }\end{array}$ \\
\hline 415 & Hancock & $\begin{array}{l}\text { German Twp., Sec. } 33 \text {. E. } \\
1 / 2 \text { of } \mathrm{Sw} .1 / 4 . \text { Near } \\
\text { west edge }\end{array}$ & $\begin{array}{l}2.5 \text { ft. brown, coarsely fi- } \\
\text { brous. } \\
15.5 \text { ft. brownish black to } \\
\text { reddish gray below. } \\
\text { Bored into a piece of wood } \\
\text { at } 18 \text { ft. and could go no } \\
\text { deeper. }\end{array}$ \\
\hline 416 & Hancock & $\begin{array}{l}\text { German Twp., on line be- } \\
\text { tween Secs. } 4 \text { and } 9 \text {, and } \\
100 \text { yds. east of edge of } \\
\text { bog }\end{array}$ & $\begin{array}{l}3.5 \text { ft. brown, fibrous. } \\
3.5 \text { ft. brownish black. }\end{array}$ \\
\hline 417 & Hancock & $\begin{array}{l}\text { German Twp., Sec. } 9 . \\
\text { Along ditch } 400 \text { yds. S. } \\
\text { sample No. } 416\end{array}$ & $\begin{array}{l}5.5 \text { ft. brown, coarsely fi- } \\
\text { brous. }\end{array}$ \\
\hline 418 & Hancock & $\begin{array}{l}\text { German Twp., on line be- } \\
\text { tween Secs. } 4 \text { and } 9 \text { at } \\
\text { middle of bog }\end{array}$ & $\begin{array}{l}7 \text { ft. brown, fibrous. } \\
9.5 \text { ft. brownish black. } \\
15.5 \text { ft. red to grayish } \\
\text { brown. B ot to m not } \\
\text { reached. }\end{array}$ \\
\hline 419 & Hancock & $\begin{array}{l}\text { German Twp., on line be- } \\
\text { tween Secs. } 4 \text { and } 9.120 \\
\text { yds. from W. edge }\end{array}$ & $4.5 \mathrm{ft}$. brown, fibrous. \\
\hline 420 & Hancock & $\begin{array}{l}\text { German and Garfield Twp. } \\
\text { line, Secs. } 4 \text { and } 33 \text { re- } \\
\text { spectively. } 100 \text { yds. } \\
\text { from island on east } \\
\text { side }\end{array}$ & $\begin{array}{l}5 \text { ft. brown, fibrous. } \\
2 \text { ft. brownish black. }\end{array}$ \\
\hline 421 & Hancock & $\begin{array}{l}\text { German and Garfield Twp. } \\
\text { line. West of sample } \\
\text { No. } 420 \text {, near middle of } \\
\text { bog }\end{array}$ & $\begin{array}{l}7 \text { ft. brown, fibrous. } \\
1.5 \text { ft. brownish black. }\end{array}$ \\
\hline
\end{tabular}


TABLE II-CONTINUED

\begin{tabular}{|c|c|c|c|}
\hline Lab.No. & County & Detailed Location of Sample & Description of Section \\
\hline 511 & Winnebago & $\begin{array}{l}\text { Center Twp., Sec. } 15 . \text { Sw. } \\
1 / 4 \text { of Ne. } 1 / 4\end{array}$ & $\begin{array}{l}9 \text { ft. brown, fibrous. } \\
4.5 \text { ft. dark brown. } \\
\text { Clayey peat at bottom. }\end{array}$ \\
\hline 514 & Winnebago & $\begin{array}{l}\text { Center Twp., Sec. } 13 . \\
\text { Northeast corner of } \mathrm{Nw} . \\
1 / 4 \text { of } \mathrm{Nw} .1 / 4\end{array}$ & $\begin{array}{l}9 \text { ft. brown, fibrous. } \\
2 \text { ft. dark brown. }\end{array}$ \\
\hline 517 & Winnebago & $\begin{array}{l}\text { Center Twp., Sec. 14. Cen- } \\
\text { ter of Se. } 1 / 4 \text { of Ne. } 1 / 4\end{array}$ & $\begin{array}{l}11 \mathrm{ft} \text {. brown, fibrous. } \\
4 \mathrm{ft} \text {. dark brown. } \\
4 \mathrm{ft} \text {. reddish brown. }\end{array}$ \\
\hline 512 & Winnebago & $\begin{array}{l}\text { Center Twp., Sec. } 35 \text {. Near } \\
\text { north line of Sec. west } \\
\text { of dredge ditch }\end{array}$ & $\begin{array}{l}9 \text { ft. brown, fibrous. } \\
2 \text { ft. fine, brown, fibrous. } \\
4 \text { ft. dark brown. }\end{array}$ \\
\hline 523 & Winnebago & $\begin{array}{l}\text { Center Twp., Sec. } 34.175 \\
\text { yds. east } 1 / 2 \text { section line } \\
\quad\end{array}$ & $\begin{array}{l}5 \mathrm{ft} \text {. brown, fibrous. } \\
6 \mathrm{ft} \text {. brown, slightly fibrous. } \\
10 \mathrm{ft} \text {. light brown. } \\
2 \mathrm{ft} \text {. reddish brown. } \\
1 \mathrm{ft} \text {. light brown. } \\
\text { Did not reach bottom. }\end{array}$ \\
\hline 524 & Winnebago & $\begin{array}{l}\text { Center 'Twp., Sec. } 34.200 \\
\text { yds. east of center }\end{array}$ & $\begin{array}{l}5 \mathrm{ft} \text {. brown, fibrous. } \\
4 \mathrm{ft} \text {. brown, slightly fibrous. } \\
14 \mathrm{ft} \text {. light brown. }\end{array}$ \\
\hline 515 & Worth & $\begin{array}{l}\text { Bristol Twp., Sec. 17. Sw, } \\
1 / 4 \text { of } \mathrm{Sw} .1 / 4225 \text { yds. } \\
\text { north of Sw. corner }\end{array}$ & $8.5 \mathrm{ft}$. brown, fibrous. \\
\hline 518 & Winnebago & $\begin{array}{l}\text { Center Twp., Sec. } 26 . \mathrm{Nw} \\
1 / 4 \text { of Se. } 1 / 4\end{array}$ & $\begin{array}{l}11 \mathrm{ft} \text {. brown, fibrous. } \\
4 \mathrm{ft.} \mathrm{brown.} \\
10 \mathrm{ft} \text {. reddish brown. } \\
\text { Did not reach bottom. }\end{array}$ \\
\hline 516 & Winnebago & $\begin{array}{l}\text { Center Twp., Sec. } 35 \text {. Se. } \\
1 / 4 \text { of Se. 1/4. Center of } \\
\text { north line }\end{array}$ & $\begin{array}{l}7 \mathrm{ft} \text {, brown, fibrous. } \\
10 \mathrm{ft} \text {. brown. }\end{array}$ \\
\hline 520 & Winnebago & $\begin{array}{l}\text { Center Twp., Sec. } 35 . \mathrm{S} \\
\text { side } 125 \text { yds. Nw. from } \\
\text { bridge over dredge ditch }\end{array}$ & $\begin{array}{l}9 \mathrm{ft} . \text { brown, fibrous. } \\
7 \mathrm{ft} \text {. brown. }\end{array}$ \\
\hline $\begin{array}{l}526 \\
\cdot\end{array}$ & Winnebago & $\begin{array}{l}\text { Mt. Valley Twp., Sec. } 2 . \\
\text { Ne. } 1 / 4 \text { of Nw, } 1 / 4 . \quad \text { E. of } \\
\text { dredge ditch, } 250 \text { yds. } \\
\text { south of road }\end{array}$ & $\begin{array}{l}11 \mathrm{ft} \text {. brown, fibrous. } \\
6 \mathrm{ft} \text {. brown. } \\
10.5 \mathrm{ft} \text {. reddish brown. }\end{array}$ \\
\hline 521 & Winnebago & $\begin{array}{l}\text { Mt. Valley Twp., Sec. } 2 . \\
\text { Nw. } 1 / 4 \text { of Sw. } \\
\text { yds. from road }\end{array}$ & $\begin{array}{l}9 \text { ft. brown, fibrous. } \\
6 \text { ft. brown. } \\
4 \text { ft. light gray. }\end{array}$ \\
\hline
\end{tabular}


TABLE II-CONTINUED

\begin{tabular}{|c|c|c|c|}
\hline$L a b . N o$ & County & Detailed Location of Sample & Description of Section \\
\hline 527 & Winnebago & $\begin{array}{l}\text { Mt. Valley Twp., Sec. } 2 . \\
\text { Se. } 1 / 4 \text { of Nw. 1/4, } 100 \\
\text { yds. W. of north and } \\
\text { south } 1 / 2 \text { section line }\end{array}$ & $\begin{array}{l}11 \mathrm{ft} \text {. brown, fibrous. } \\
2 \mathrm{ft.} \mathrm{black.} \\
4 \mathrm{ft} \text {. brown. }\end{array}$ \\
\hline 528 & Winnebago & $\begin{array}{l}\text { Mt. Valley Twp., Sec. } 2 \text {. } \\
\text { Sw. } 1 / 4 \text { of Ne. 1/4, } 175 \\
\text { yds. E. of north and } \\
\text { south } 1 / 2 \text { section line }\end{array}$ & $\begin{array}{l}11 \mathrm{ft} \text {. brown, flbrous. } \\
6 \mathrm{ft} \text {. brown. } \\
6 \mathrm{ft} \text {. reddish brown. } \\
3 \mathrm{ft} \text {. brown. }\end{array}$ \\
\hline 525 & Winnebago & $\begin{array}{l}\text { Center Twp., Sec. } 25 . \text { Ne. } \\
1 / 4 \text { of Ne. } 1 / 4 \text {, near } W . \\
\text { line }\end{array}$ & $\begin{array}{l}9 \text { ft. brown, fibrous. } \\
4 \text { ft. black. }\end{array}$ \\
\hline 529 & Winnebago & $\begin{array}{l}\text { Center } T w p_{.,} \text {Sec. } 24 . S w . \\
1 / 4 \text { of } S \text {. } 1 / 4 \text { near center }\end{array}$ & $\begin{array}{l}11 \text { ft. brown, fibrous. } \\
6 \mathrm{ft} \text {. dark brown. } \\
2 \text { ft. reddish brown. }\end{array}$ \\
\hline 530 & Winnebago & $\begin{array}{l}\text { Center Twp., Sec. 24. Se. } \\
1 / 4 \text { of Se. } 1 / 4,100 \text { yds. } \\
\text { S. of Ne. corner }\end{array}$ & $\begin{array}{l}11 \mathrm{ft} \text {. brown, fibrous. } \\
4 \mathrm{ft} \text {. brown. } \\
11 \mathrm{ft} \text {. reddish brown. }\end{array}$ \\
\hline 513 & Worth & $\begin{array}{cll}\text { Bristol } & \text { Twp., } & \text { Sec. } 20.300 \\
\text { yds. Nw. from road } & \text { rom } \\
\text { along } & \text { dredge ditch }\end{array}$ & $\begin{array}{l}9 \text { ft. brown, fibrous. } \\
1 \text { ft. brown. }\end{array}$ \\
\hline 519 & Worth & $\begin{array}{l}\text { Bristol Twp., Sec. } 28 \text {. S. } \\
\text { side Sec. } 150 \text { yds. from } \\
\text { road, and } 250 \text { yds. from } \\
\text { W. line }\end{array}$ & $\begin{array}{l}9 \text { ft. dark brown (somewhat } \\
\text { fibrous clayey peat un- } \\
\text { derlying above). }\end{array}$ \\
\hline 522 & Worth & $\begin{array}{c}\text { Bristol Twp., Sec. 28. } 200 \\
\text { yds. due N. of No. } 519 \\
\text { • }\end{array}$ & $\begin{array}{l}5 \mathrm{ft} \text {. dark brown, somewhat } \\
\text { fibrous. } \\
4 \mathrm{ft} \text {. dark brown. } \\
2 \mathrm{ft} \text {. reddish brown. }\end{array}$ \\
\hline 531 & Worth & $\begin{array}{l}\text { Bristol Twp., Sec. } 27.125 \\
\text { yds. Nw. of center of } \\
\text { Sw. } 1 / 4\end{array}$ & $\begin{array}{l}5 \text { ft. dark brown, somewhat } \\
\text { fibrous. } \\
7 \text { ft. dark brown. }\end{array}$ \\
\hline 532 & Worth & $\begin{array}{l}\text { Bristol Twp., Sec. } 20 \text {. Sw. } \\
1 / 4 \text {, near middle }\end{array}$ & 9 ft. brown, fibrous. \\
\hline 538 & Winnebago & $\begin{array}{l}\text { Center Twp., Sec. 14. Se. } \\
1 / 4 \text { of } \mathrm{Sw} .1 / 4 \text {, near } \mathrm{Nw} . \\
\text { corner }\end{array}$ & $\begin{array}{l}9 \mathrm{ft} \text { brown, fibrous. } \\
6 \mathrm{ft} \text { dark brown. } \\
7 \mathrm{ft} \text { reddish brown. }\end{array}$ \\
\hline 539 & Winnebago & $\begin{array}{l}\text { Center Twp., Sec. } 14 . \text { Se. } \\
1 / 4 \text { of } S w .1 / 4 \text {, near } N w \\
\text { corner }\end{array}$ & $\begin{array}{l}6.5 \text { ft. dark brown, slightly } \\
\text { fibrous. }\end{array}$ \\
\hline 540 & Winnebago & $\begin{array}{c}\text { Center Twp., Sec. } 24 \text {. Nw. } \\
1 / 4 \text { of Se. } 1 / 4,200 \text { yds. } \\
\text { Ne. from Sw. corner }\end{array}$ & $\begin{array}{l}9 \text { ft. brown, fibrous. } \\
2 \text { ft. dark brown. }\end{array}$ \\
\hline
\end{tabular}


TABLE II-CONTINUED

\begin{tabular}{|c|c|c|c|}
\hline Lab.No. & County & Detailed Location of Sample & Description of Section \\
\hline 541 & Winnebago & $\begin{array}{l}\text { Center Twp., Sec. } 24 . \text { Cen- } \\
\text { ter } \mathrm{Nw} .1 / 4\end{array}$ & $\begin{array}{l}9 \text { ft. brown, fibrous. } \\
7 \text { ft. brown, slightly fibrous. }\end{array}$ \\
\hline 533 & Worth & $\begin{array}{l}\text { Hartland Twp., Sec. } 29 . \\
\text { Ne. } 1 / 4 \text { of } \mathrm{Nw} .1 / 4 \text {, near } \\
\text { Ne. corner }\end{array}$ & 9 ft. brown, fibrous. \\
\hline 544 & Winnebago & $\begin{array}{l}\text { Linden Twp., Sec. } 22 . \\
\text { Near middle north line }\end{array}$ & $\begin{array}{l}5 \text { ft. brown, fibrous. } \\
2 \text { ft. brown, slightly flbrous. } \\
6 \text { ft. black. }\end{array}$ \\
\hline 545 & Winnebago & $\begin{array}{l}\text { Linden Twp., Sec. } 14.100 \\
\text { yds. S. of center of } \mathrm{Sw} . \\
1 / 4 \text { of } \mathrm{Sw} .1 / 4\end{array}$ & 5 ft. brown, fibrous. \\
\hline 546 & Winnebago & $\begin{array}{l}\text { Linden Twp., Sec. } 14 . \text { Se. } \\
\text { corner of Sw. } 1 / 4 \text { of Sw. } \\
1 / 4\end{array}$ & $\begin{array}{l}4.5 \mathrm{ft} \text { brown, fibrous, chang- } \\
\text { ing to black. }\end{array}$ \\
\hline 556 & Winnebago & $\begin{array}{c}\text { King Twp., Sec. } 22 . \text { Cen- } \\
\text { ter of Sw. } 1 / 4 \text { of } \mathrm{Ne} .1 / 4\end{array}$ & $\begin{array}{l}5 \text { ft. brown, fibrous. } \\
3 \text { ft. dark brown. } \\
4 \text { ft. black. }\end{array}$ \\
\hline 542 & Winnebago & $\begin{array}{l}\text { Linden Twp., Sec. } 29 . \text { Cen- } \\
\text { ter of } N \dot{w} .1 / 4 \text { of Se. } 1 / 4\end{array}$ & $\begin{array}{l}5 \text { ft. brown, fibrous. } \\
2 \text { ft. dark brown. } \\
2 \text { ft. black. }\end{array}$ \\
\hline 547 & Winnebago & $\begin{array}{c}\text { Logan Twp., Sec. } 24.150 \\
\text { yds. Ne. from Sw. cor- } \\
\text { ner of Sw. } 1 / 4 \text { of } \mathrm{Sw} .1 / 4\end{array}$ & $\begin{array}{l}9 \mathrm{ft} \text {. brown, fibrous. } \\
15 \mathrm{ft} \text {. brown. } \\
\text { Did not reach bottom o? } \\
\text { peat. }\end{array}$ \\
\hline 534 & Winnebago & $\begin{array}{l}\text { Logan Twp., Sec. 24. Cen- } \\
\text { ter of Sw. 1/4 }\end{array}$ & $\begin{array}{l}7 \text { ft. brown, fibrous. } \\
2 \text { ft. black. } \\
\text { Gravel bottom. }\end{array}$ \\
\hline 535 & Winnebago & $\begin{array}{l}\text { Logan Twp., Sec. } 23 . \quad 150 \\
\text { yds. Sw. of center }\end{array}$ & $\begin{array}{l}5 \text { ft. brown, fibrous. } \\
1 \text { ft. black. }\end{array}$ \\
\hline 536 & Winnebago & $\begin{array}{l}\text { Logan Twp., Sec. } 23.200 \\
\text { yds. Nw. of center }\end{array}$ & $\begin{array}{l}6 \text { ft. brown, fibrous. } \\
1 \text { ft. black. }\end{array}$ \\
\hline 537 & Winnebago & $\begin{array}{l}\text { Logan Twp., Sec. } 23 . \text { Nw. } \\
1 / 4,200 \text { yds. from road }\end{array}$ & $\begin{array}{l}8.5 \mathrm{ft} \text {. dark brown, slightly } \\
\text { fibrous. } \\
\text { Gravel bottom. }\end{array}$ \\
\hline 550 & Hancock & $\begin{array}{l}\text { Crystal Twp., Sec. 2. Near } \\
\text { Sec. line, } 100 \text { yds. from } \\
\text { W. edge of bog }\end{array}$ & $\begin{array}{l}5 \text { ft. brown, fibrous. } \\
9 \text { ft. brown, } \\
\text { Peat mud continues. }\end{array}$ \\
\hline 548 & Hancock & $\begin{array}{c}\text { Crystal Twp., Sec. 2. On } \\
1 / 4 \text { Sec. line east side of } \\
\text { bog near section line }\end{array}$ & $\begin{array}{l}5 \text { ft. brown, fibrous. } \\
4 \text { ft. brown, gradually be } \\
\text { coming poorer. }\end{array}$ \\
\hline
\end{tabular}


TABLE II-CONTINUED

\begin{tabular}{|c|c|c|c|}
\hline Lab. No. & County & Detailed Location of Sample & Description of Section \\
\hline 549 & Winnebago & $\begin{array}{l}\text { Mt. Valley Twp., Sec. } 33 . \\
\text { Nw. } 1 / 4 \text { of Nw. } 1 / 4\end{array}$ & 5.5 ft. brown, fibrous. \\
\hline 575 & Winnebago & $\begin{array}{l}\text { Mt. Valley Twp., Sec. } 33 . \\
300 \text { yds. E. and } 300 \text { yds. } \\
\text { S. of Nw. corner }\end{array}$ & $\begin{array}{l}24 \mathrm{ft} \text {. brown, fibrous peat, } \\
\text { changing to slightly fi- } \\
\text { brous. }\end{array}$ \\
\hline 551 & Winnebago & $\begin{array}{l}\text { Mt. Valley Twp., Sec. } 33 . \\
250 \text { yds. N. and } 50 \text { yds. } \\
\text { E. of No. } 575\end{array}$ & $\begin{array}{l}7 \text { ft. dark brown, fibrous. } \\
7 \text { ft. brown, slightly fibrous. }\end{array}$ \\
\hline 552 & Winnebago & $\begin{array}{l}\text { Mt. Valley Twp., Sec. } 32 . \\
\text { Nw. } 1 / 4 \text { of Nw. } 1 / 4,100 \\
\text { yds. west and } 150 \text { yds. } \\
\text { S. of Ne. corner }\end{array}$ & $\begin{array}{l}11 \mathrm{ft} . \text { brown, fibrous, chang- } \\
\text { ing to slightly fibrous. }\end{array}$ \\
\hline 553 & Winnebago & $\begin{array}{l}\text { Mt. Valley Twp., Sec. } 29 . \\
\text { Se. } 1 / 4 \text { of Se. } 1 / 4,150 \text { yds. } \\
\text { from road and midway } \\
\text { between quarter and } \\
\text { section lines }\end{array}$ & $\begin{array}{l}15 \mathrm{ft} \text {. brown, fibrous, chang- } \\
\text { ing to slightly fibrous. }\end{array}$ \\
\hline 554 & Winnebago & $\begin{array}{l}\text { Mt. Valley } \text { Twp., Sec. } 29 . \\
125 \text { yds. N. of sample } \\
\text { No. } 553\end{array}$ & $\begin{array}{l}24 \mathrm{ft} \text {. brown, fibrous, chang- } \\
\text { ing to slightly fibrous. }\end{array}$ \\
\hline 555 & Hancock & $\begin{array}{l}\text { Crystal Twp., Sec. 14. Ne. } \\
1 / 4 \text {, near north edge of } \\
\text { bog }\end{array}$ & $\begin{array}{l}6 \mathrm{ft} \text {. brown, fibrous. } \\
3 \mathrm{ft} \text { black. }\end{array}$ \\
\hline 576 & Hancock & $\begin{array}{l}\text { Garfield Twp., Sec. 23. } 200 \\
\text { yds. S. and } 100 \text { E. of } \\
\text { Nw. corner }\end{array}$ & $5 \mathrm{ft}$. brown, fibrous. \\
\hline 577 & Hancock & $\begin{array}{c}\text { Garfield Twp., Sec. } 23.300 \\
\text { yds. south of No. } 576\end{array}$ & $\begin{array}{l}9 \mathrm{ft} \text { brown, fibrous. } \\
3 \text { ft. brown, somewhat } \\
\text { fibrous. } \\
\text { A peat mud continues } 14 \mathrm{ft} \text {. }\end{array}$ \\
\hline 578 & Hancock & $\begin{array}{c}\text { Garfield Twp., Sec. } 23.300 \\
\text { yds. south of No. } 577\end{array}$ & $\begin{array}{l}9 \text { ft. brown, fibrous. } \\
3 \text { ft. fine, brown, somewhat } \\
\text { fibrous. }\end{array}$ \\
\hline 579 & Hancock & $\begin{array}{l}\text { Garfield Twp., Sec. } 23 . \\
\text { Near center of Sw. } 1 / 4 \\
\text { of Sw. } 1 / 4\end{array}$ & $\begin{array}{l}5 \text { ft. brown, fibrous. } \\
1 \text { ft. brown. }\end{array}$ \\
\hline 580 & Hancock & $\begin{array}{l}\text { Garfield Twp., Sec. 18. On } \\
\text { quarter section line }\end{array}$ & $\begin{array}{l}9 \text { ft. brown, fibrous. } \\
4 \text { ft. black. }\end{array}$ \\
\hline 581 & Hancock & $\begin{array}{l}\text { Garfield Twp., Sec. } 18.175 \\
\text { yds. directly } \\
580\end{array}$ & $5 \mathrm{ft}$ brown, fibrous. \\
\hline
\end{tabular}


TABLE II-CONTINUED

\begin{tabular}{|c|c|c|c|}
\hline Lab. No. & County & Detailed Location of Sample & Descruption of Section \\
\hline 584 & Cerro Gordo & $\begin{array}{l}\text { Lake Twp., Sec. } 19.100 \\
\text { yds. S. and } 100 \text { yds. W. } \\
\text { of Ne. corner Ne. } 1 / 4 \text { of } \\
\text { Sw. } 1 / 4\end{array}$ & $\begin{array}{l}14 \text { ft. brown, fibrous, grad- } \\
\text { ually changing to less } \\
\text { fibrous. }\end{array}$ \\
\hline$\cdot 597$ & Cerro Gordo & $\begin{array}{l}\text { Lake Twp., Sec. } 19 . \mathrm{Nw} . \\
1 / 4 \text { of } \mathrm{Sw} .1 / 4,75 \mathrm{yds} . \mathrm{S} . \\
\text { of center }\end{array}$ & $4.5 \mathrm{ft}$. brown, fibrous. \\
\hline 585 & Cerro Gordo & $\begin{array}{l}\text { Lake Twp., Sec. 19. } 220 \\
\text { yds. from S. line and } \\
125 \text { yds. from W. edge } \\
\text { of bog }\end{array}$ & $\begin{array}{l}9 \mathrm{ft} \text {. brown, fibrous, chang- } \\
\text { ing to slightly fibrous. }\end{array}$ \\
\hline 586 & Cerro Gordo & Lake Twp., Sec. 19 & 7 ft. brown, fibrous. \\
\hline 583 & Cerro Gordo & $\begin{array}{l}\text { Lake Twp., on line be- } \\
\text { tween Secs. } 29 \text { and } 30 . \\
\text { near south side }\end{array}$ & 4 ft. brown, fibrous. \\
\hline 587 & Cerro Gordo & $\begin{array}{r}\text { Lake Twp., Sec. } 29 . \text { Cen- } \\
\text { ter Nw. 1/4 of Sw. 1/4 }\end{array}$ & $\begin{array}{l}7 \text { ft. brown, fibrous, grad. } \\
\text { ually changing to less } \\
\text { fibrous. }\end{array}$ \\
\hline 598 & Cerro Gordo & $\begin{array}{l}\text { Lake Twp., Sec. 29. Near } \\
\text { middle north line of } \mathrm{Sw} \text {. } \\
1 / 4 \text { of } \mathrm{Sw} .1 / 4\end{array}$ & $\begin{array}{l}7 \mathrm{ft} \text {. brown, fibrous, grad- } \\
\text { ually changing to less } \\
\text { fibrous. }\end{array}$ \\
\hline 591 & Cerro Gordo & $\begin{array}{l}\text { Lake Twp., Sec. } 29 \text {. South } \\
\text { side at center of neck } \\
\text { of bog where it crosses } \\
\text { road }\end{array}$ & 5 ft. brown, fibrous. \\
\hline 592 & Cerro Gordo & $\begin{array}{l}\text { Mt. Vernon Twp., section } \\
\text { line between } 5 \text { and } 8 \\
\text { near middle }\end{array}$ & $5 \mathrm{ft}$. brown, fibrous. \\
\hline 593 & Cerro Gordo & $\begin{array}{l}\text { Mt. Vernon Twp. } 225 \text { yds. } \\
\text { due west of No. } 592\end{array}$ & 5 ft. brown, fibrous. \\
\hline 596 & Cerro Gordo & $\begin{array}{c}\text { Mt. Vernon Twp., Sec. } 8 \\
300 \text { yds. Se. of No. } 593\end{array}$ & 4 ft. brown, fibrous. \\
\hline 594 & Celro Gordo & $\begin{array}{l}\text { Clear Lake Twp., Sec. } 35 . \\
\text { Near center Sw. } 1 / 4 \text { of } \\
\text { Sw. } 1 / 4\end{array}$ & $\begin{array}{l}7 \text { ft. brown, fibrous, grad- } \\
\text { uaily changing to less } \\
\text { fibrous. }\end{array}$ \\
\hline 599 & Cerro Gordo & $\begin{array}{l}\text { Union Twp., Sec. } 2 . \text { Near } \\
\text { center Nw. } 1 / 4 \text { of } \mathrm{Nw} .1 / 4\end{array}$ & $\begin{array}{l}8 \text { ft. brown, fibrous. } \\
\text { Peat mud continues to a } \\
\text { greater depth. }\end{array}$ \\
\hline 600 & Cerro Gordo & $\begin{array}{l}\text { Union Twp.. Sec. 2. } 175 \\
\text { yds. Ne. No. } 599\end{array}$ & 3.5 ft. brown, fibrous. \\
\hline
\end{tabular}


TABLE II-CONTINUED

\begin{tabular}{|c|c|c|c|}
\hline Lab. No. & inty & Detailed Location of Sample & Description of Section \\
\hline 601 & Cerro Gordo & $\begin{array}{l}\text { Union Twp., Sec. } 2 . \quad 150 \\
\text { yds. Se. of Nw. corner } \\
\text { of Ne. } 1 / 4\end{array}$ & $\begin{array}{l}5 \mathrm{ft} \text {. brown, fibrous. } \\
\text {. }\end{array}$ \\
\hline 602 & Cerro Gordo & $\begin{array}{l}\text { On Union-Clear Lake Twp. } \\
\text { line, between sections } 2 \\
\text { and } 35.200 \text { yds. west } \\
\text { of middle }\end{array}$ & 5 ft. brown, fibrous. \\
\hline 614 & Cerro Gordo & $\begin{array}{l}\text { Union Twp. } 200 \text { yds. W. } \\
\text { of center of Sec. } 12\end{array}$ & 5 ft. brown, fibrous. \\
\hline 615 & Cerro Gordo & $\begin{array}{l}\text { Tnion Twp. Sw. } 1 / 4 \text { of } \\
\mathrm{Nw} .1 / 4 \text { of Sec. } 12\end{array}$ & $\begin{array}{l}5 \text { ft. brown, fibrous, low } \\
\text { grade. }\end{array}$ \\
\hline 616 & Cerro Gordo & $\begin{array}{l}\text { Union Twp., Sec. } 11 \text {. Se. } \\
1 / 4 \text { of Se. } 1 / 4 \text {, near center }\end{array}$ & $7 \mathrm{ft}$. brown, fibrous. \\
\hline 617 & Cerro Gordo & $\begin{array}{l}\text { Union Twp., Sec. 12. Sw. } \\
1 / 4 \text { of Sw. } 1 / 4 \text {, near center }\end{array}$ & $\begin{array}{l}7 \mathrm{ft} \text { brown, fibrous. } \\
\text { Low grade peat continues } \\
\text { down to } 12 \mathrm{ft} \text {. }\end{array}$ \\
\hline 618 & Cerro Gordo & $\begin{array}{l}\text { Union Twp. Center of } \\
\text { neck of bog on north } \\
\text { line, } 220 \text { yds. from road } \\
\text { on east line }\end{array}$ & 4 et. brown, fibrous. \\
\hline 619 & Cerro Gordo & $\begin{array}{l}\text { Union Twp. Near center } \\
\text { of Se. quarter }\end{array}$ & $8 \mathrm{ft}$ brown, fibrous. \\
\hline 620 & Cerro Gordo & $\begin{array}{l}\text { Union Twp. On } 1 / 2 \text { Sec. } \\
\text { line near road on east }\end{array}$ & $\begin{array}{l}6 \mathrm{ft} \text {, brown, fibrous, under- } \\
\text { laid with blue clay. }\end{array}$ \\
\hline 621 & Kossuth & Ramsay Twp., Sec. 26. & $\begin{array}{l}4 \text { ft. brown. fibrous, chang- } \\
\text { ing to black peaty mud. }\end{array}$ \\
\hline 622 & .Kossuth & $\begin{array}{l}\text { Ramsay Twp.. Sec. } 26 . \\
\text { North side near center } \\
\text { of bog }\end{array}$ & $\begin{array}{l}2.5 \mathrm{ft} \text {. brown, fibrous. } \\
? .5 \mathrm{ft} \text { black peaty mud. } \\
\text { Pentv mud continues below } \\
8 \mathrm{ft} \text {. }\end{array}$ \\
\hline 623 & Kossuth & $\begin{array}{l}\text { Ramsay Twp., Sec. } 35.250 \\
\text { yds. Nw. of center of } \\
\text { section and at center of } \\
\text { bog }\end{array}$ & $\begin{array}{l}2 \mathrm{ft} . \text { brown, fibrous. } \\
2 \mathrm{ft} . \text { black peaty mud. }\end{array}$ \\
\hline 624 & Kossuth & $\begin{array}{l}\text { Greenwood Twp., Sec. } 11 . \\
\text { Ne. corner of bog, } 100 \\
\text { yds. west on } 1 / 4 \text { Sec. line }\end{array}$ & $\begin{array}{l}5 \text { ft. brown, fibrous, grad- } \\
\text { ing downward into black } \\
\text { peaty mud. }\end{array}$ \\
\hline 625 & Kossuth & $\begin{array}{l}\text { Greenwood Twp., Sec. 11. } \\
100 \text { yds. west of sample } \\
\text { No. } 624\end{array}$ & $\begin{array}{l}6 \mathrm{ft} \text {. brown. fibrous, grad- } \\
\text { ing downward into black } \\
\text { peaty mud. }\end{array}$ \\
\hline
\end{tabular}


TABLE II-CUNTINUED

\begin{tabular}{|c|c|c|c|}
\hline Lab.No. & County & Detailed Location of Sample & Description of Section \\
\hline 626 & Kossuth & $\begin{array}{l}\text { Greenwood Twp., Sec. 11. } \\
\text { At center of bog }\end{array}$ & $\begin{array}{l}6.5 \text { ft. brown, fibrous, grad- } \\
\text { ing downward into black } \\
\text { peaty mud. }\end{array}$ \\
\hline $634 \mathrm{P}$ & Kossuth & $\begin{array}{l}\text { Sherman Twp., Sec. } 14 . \\
\text { Sw. cormer, } 50 \text { yds. N. } \\
\text { and } 275 \text { yds. E, of Sec. } \\
\text { line }\end{array}$ & $\begin{array}{l}3 \text { ft. brown, fibrous. } \\
3 \text { ft. black peaty mud. }\end{array}$ \\
\hline $635 \mathrm{P}$ & Kossuth & $\begin{array}{l}\text { Sherman Twp., Sec. } 23 . \\
150 \mathrm{yds} \text {. Nw. of center } \\
\text { of Sec. }\end{array}$ & $\begin{array}{l}3 \text { ft. brown, fibrous. } \\
2 \text { ft. black peaty mud. }\end{array}$ \\
\hline $636 \mathrm{P}$ & Kossuth & $\begin{array}{l}\text { Sherman Twp., Sec. } 26 . \\
325 \text { yds. west from } \mathrm{E} . \\
\text { line and } 200 \text { yds. N. of } \\
1 / 4 \text { line }\end{array}$ & $\begin{array}{l}4 \text { ft. brown, fibrous. } \\
1 \text { ft. dark brown. }\end{array}$ \\
\hline $637 \mathrm{P}$ & Kossuth & $\begin{array}{l}\text { Sherman Twp., Sec. } 26 . \text { On } \\
\text { east line near middle }\end{array}$ & $4 \mathrm{ft}$. brown, fibrous. \\
\hline $638 \mathrm{P}$ & Kossuth & $\begin{array}{l}\text { Sherman Twp., Sec, } 25 . \\
\text { Middle of neck of bog } \\
\text { in Sw. } 1 / 4\end{array}$ & 4 ft. brown, fibrous. \\
\hline $639 \mathrm{P}$ & Kossuth & $\begin{array}{l}\text { Sherman Twp., Sec. } 26 . \\
\text { Near bridge on north } \\
\text { line road }\end{array}$ & 5 ft. brown, fibrous. \\
\hline $640 \mathrm{P}$ & Clay & $\begin{array}{l}\text { Freeman Twp.' Sec. } 23 . \\
250 \text { yds. Nw. center of } \\
\text { Sec. }\end{array}$ & $\begin{array}{l}4 \text { ft. brown, fibrous. Be- } \\
\text { comes darker below. }\end{array}$ \\
\hline $641 \mathrm{P}$ & Clay & $\begin{array}{l}\text { Freeman Twp., Sec. } 23 . \\
\text { Center of } \mathrm{Nw}, 1 / 4\end{array}$ & 4 ft. brown, fibrous. \\
\hline $642 \mathrm{P}$ & Clay & $\begin{array}{l}\text { Freeman Twp., Sec. } 11 . \\
\quad \text { Near middle on S. line }\end{array}$ & $\begin{array}{l}4 \text { ft. brown, fibrous. Very } \\
\text { soft below. }\end{array}$ \\
\hline $643 \mathrm{P}$ & Palo Alto & $\begin{array}{l}\text { Lost Island Twp., Sec. } 11 . \\
125 \text { yds. from N. edge } \\
\text { of bog }\end{array}$ & 3 ft. brown, fibrous. \\
\hline $644 \mathrm{P}$ & Palo Alto & $\begin{array}{l}\text { Lost Island Twp., Sec. } 11 . \\
200 \text { yds. E. of sample } \\
\text { No. } 643 \mathrm{P}\end{array}$ & 3 ft. brown, fibrous. \\
\hline $645 \mathrm{P}$ & Palo Alto & $\begin{array}{l}\text { Lost Island Twp., Sec. } 11 . \\
\text { Se. 1/4, } 440 \text { yds. S. of } \\
\text { lake and } 150 \text { yds. from } \\
\text { road }\end{array}$ & 3 ft. brown, fibrous. \\
\hline 638 & Dickinson & $\begin{array}{c}\text { Richland Twp., Sec. } 17 . \\
\text { Ne. 1/4 of Se. 1/4 near } \\
\text { center of bog }\end{array}$ & 3 ft. brown, fibrous. \\
\hline
\end{tabular}


TABLE II-CONTINUED

\begin{tabular}{|c|c|c|c|}
\hline$L a b . N o$ & County & Detailed Location of Sample & Description of Section \\
\hline 639 & Wright & $\begin{array}{l}\text { Belmond Twp., Sec. } 17 . \\
300 \text { yds. S. of Ne. cor- } \\
\text { ner }\end{array}$ & 5 ft. brown, fibrous. \\
\hline 640 & Hancock & $\begin{array}{l}\text { Twin Lake Twp., Sec. } 32 . \\
\text { On E. and W. Sec. line } \\
\text { and } 100 \text { yds. from edge } \\
\text { of bog }\end{array}$ & $4.5 \mathrm{ft}$. brown, fibrous. \\
\hline 641 & Hancock & $\begin{array}{l}\text { Twin Lake Twp., Sec. } 32 . \\
100 \text { yds. N. of S. line of } \\
\text { Sec. and } 125 \text { yds. from } \\
\text { E. edge of bog }\end{array}$ & $\begin{array}{c}4.5 \mathrm{ft} \text {. brown, fibrous. } \\
\text {. }\end{array}$ \\
\hline 422 & Hancock & $\begin{array}{l}\text { German and Garfield Twp. } \\
\text { line. } 150 \mathrm{yds} \text {. E. of W. } \\
\text { ditch }\end{array}$ & $\begin{array}{l}8.5 \text { ft. brown, fibrous. } \\
4 \text { ft. brownish black. } \\
6 \text { ft. reddish brown. }\end{array}$ \\
\hline 423 & Hancock & $\begin{array}{c}\text { German \& Garfield Twp. } \\
\text { line. On west ditch }\end{array}$ & $\begin{array}{l}8.5 \text { ft. brown, fibrous. } \\
2.5 \text { ft. brownish black. } \\
6 \text { ft. lighter brown. }\end{array}$ \\
\hline 424 & Worth & $\begin{array}{l}\text { Fertile Twp., Sec. 19. Se. } \\
\text { 1/4 near S. line }\end{array}$ & $\begin{array}{l}6 \mathrm{ft} \text {. brown, fibrous. } \\
14 \mathrm{ft} \text {. brownish black. }\end{array}$ \\
\hline 425 & Worth & $\begin{array}{l}\text { Fertile Twp., Sec. } 19.150 \\
\text { yds. E. of N. of sample } \\
\text { No. } 424\end{array}$ & $\begin{array}{l}10 \text { ft. brown, fibrous, dark- } \\
\text { er below. } \\
1 \text { ft. marly peat. } \\
5 \text { ft. brownish black. }\end{array}$ \\
\hline 426 & Worth & $\begin{array}{l}\text { Fertile Twp., Sec. } 19.200 \\
\text { yds. E. of N. of sample } \\
\text { No. } 425\end{array}$ & $\begin{array}{l}12 \text { ft. brown, fibrous, dark } \\
\text { er below. } \\
4 \text { ft. marly peat. } \\
3 \text { ft. brownish peat, hard. }\end{array}$ \\
\hline 427 & Worth & $\begin{array}{l}\text { Silver Lake Twp., on line } \\
\text { of Secs. } 13 \text { and } 14 . \text { At } \\
\text { south bridge }\end{array}$ & $\begin{array}{l}25 \mathrm{ft} \text { brown, fibrous, becom } \\
\text { ing darker and less } \\
\text { fibrous below. } \\
\text { Did not reach bottom. }\end{array}$ \\
\hline 428 & Winnebago & $\begin{array}{l}\text { Mt. Valley Twp., Sec. } 25 . \\
\text { Near middle south line }\end{array}$ & $\begin{array}{l}7 \text { ft. brown, fibrous. } \\
7 \text { ft. brownish black. } \\
5 \text { ft. soft, non-fibrous peaty } \\
\text { mud. }\end{array}$ \\
\hline 429 & Worth & $\begin{array}{l}\text { Silver Lake Twp., on line } \\
\text { Secs. } 13 \text { and } 14 . \\
\text { north bridge }\end{array}$ & $\begin{array}{l}\text { Same as sample No. } 427 \text {. } \\
\text { Reached grit at } 19 \mathrm{ft} .\end{array}$ \\
\hline$\overline{469}$ & Wright & $\begin{array}{l}\text { Belmond Twp., Sec. } 16 . \\
\text { Near north edge on east } \\
\text { line }\end{array}$ & $\begin{array}{l}4.5 \mathrm{ft} \text {. brown, fibrous. } \\
3.5 \mathrm{ft} \text { brownish black. }\end{array}$ \\
\hline 470 & Wright & $\begin{array}{l}\text { Belmond Twp., Sec. } 16 . \\
375 \text { yds. S. of sample } \\
\text { No. } 469\end{array}$ & $\begin{array}{l}5 \mathrm{ft} \text {. brown, fibrous. } \\
5.5 \mathrm{ft} \text {. brownish black. }\end{array}$ \\
\hline
\end{tabular}


TABLE II-CONTXNUED

\begin{tabular}{|c|c|c|c|}
\hline Lab. No. & County & Detailed Location of Sample & Description of Section \\
\hline 471 & Wright & $\begin{array}{l}\text { Belmond Twp., on line } \\
\text { Secs. } 7 \text { and } 8,150 \text { yds. } \\
\text { from S, edge bog }\end{array}$ & $\begin{array}{l}3.5 \mathrm{ft} \text {. brown, fibrous. } \\
8.5 \mathrm{ft} \text { light brown. } \\
.5 \mathrm{ft} \text {. peaty mud. } \\
5.5 \mathrm{ft} \text { reddish brown. } \\
\text { Did not reach bottom. }\end{array}$ \\
\hline 472 & Wright & $\begin{array}{l}\text { Belmond Twp., on line } \\
\text { Secs. } 7 \text { and } 8 \text {; middle of } \\
\text { bog. N. of sample No. } \\
471\end{array}$ & $\begin{array}{l}4 \text { ft. brown, fibrous. } \\
7 \text { ft. brown peat. } \\
\text { Gravel below. }\end{array}$ \\
\hline 473 & Wright & $\begin{array}{l}\text { Belmond Twp., Sec. } 7 . \text { Ne. } \\
1 / 4 \text { near road }\end{array}$ & $2.5 \mathrm{ft}$. brown, fibrous. \\
\hline 474 & Hancock & $\begin{array}{l}\text { German Twp., Sec. } 5 . \\
\text { yds. S. of middle line } \\
\text { and } 100 \text { yds. from } \\
\text { side of. bog }\end{array}$ & $\begin{array}{l}4 \mathrm{ft} \text {. brown, fibrous. } \\
1.5 \mathrm{ft} \text {. brownish black. }\end{array}$ \\
\hline $\begin{array}{r}475 \\
-\end{array}$ & Hancock & $\begin{array}{l}\text { German Twp., Sec. } 5 \text {. Mid- } \\
\text { dle of bog west of sam- } \\
\text { ple No. } 474\end{array}$ & $\begin{array}{l}4 \text { ft. brown, fibrous. } \\
3 \text { ft. brownish black. }\end{array}$ \\
\hline 476 & Hancock & $\begin{array}{c}\text { German Twp., Sec. } 5 \text {. Near } \\
\text { west edge of bog in line } \\
\text { with No. } 474 \text { and } 475\end{array}$ & $\begin{array}{l}3 \mathrm{ft} \text {. brown, fibrous. } \\
1.5 \mathrm{ft} \text {. brownish black. }\end{array}$ \\
\hline 477 & Wright & Iowa Twp., Sec. 23 & $\begin{array}{l}\text { The peaty marl from the } \\
\text { lower portion of No. } 388 .\end{array}$ \\
\hline 478 & Cerro Gordo & $\begin{array}{l}\text { Grimes Twp., Sec. } 3 . \\
\text { Along drain and due N. } \\
\text { sample No. } 404\end{array}$ & $\begin{array}{l}3.5 \mathrm{ft} \text {. brown, fibrous. } \\
8 \mathrm{ft} \text {. brownish black, grad- } \\
\text { ing into gray. } \\
13 \mathrm{ft} \text {. reddish brown. }\end{array}$ \\
\hline 479 & Cerro Gordo & Union Twp., Sec. 34, W 1/2 & $\begin{array}{l}5.5 \mathrm{ft} \text {. brown, fibrous. } \\
4 \mathrm{ft} \text {. brown. } \\
1.5 \mathrm{ft} \text {. reddish brown. } \\
8 \mathrm{ft.} \text { grayish brown, marly. }\end{array}$ \\
\hline $4 S 4$ & Hancock & $\begin{array}{l}\text { German Twp., Sec. } 33.100 \\
\text { yds. due west of sample } \\
\text { No. } 414\end{array}$ & $\begin{array}{l}2.5 \mathrm{ft} . \text { brown, fibrous. } \\
5.5 \mathrm{ft} \text {. brownish black. } \\
3 \mathrm{ft.} \text { grayish brown, marly. } \\
7 \mathrm{ft} \text {. reddish brown. }\end{array}$ \\
\hline 485 & Worth & $\begin{array}{l}\text { Silver Lake Twp.. Sec. } 22 . \\
600 \text { yds. } \\
\text { and } 150 \text { yds. } \\
\text { bridge }\end{array}$ & $\begin{array}{l}3 \text { ft. brown, fibrous. } \\
2 \text { ft. brownisli black. } \\
6 \text { ft. brown to light brown } \\
\text { below. }\end{array}$ \\
\hline 486 & Worth & $\begin{array}{l}\text { Silver Lake Twp., Sec. } 10 . \\
100 \text { yds. E. and } 200 \mathrm{~S} \text {. of } \\
\text { Nw. corner of Se. } 1 / 4 \text { of } \\
\text { Se. } 1 / 4\end{array}$ & $\begin{array}{l}5.5 \text { ft. brownish black, } \\
\text { fibrous above. } \\
\text { No. } 486 \text { is lower part only. }\end{array}$ \\
\hline
\end{tabular}


TABLE II-CoNTINUED

\begin{tabular}{|c|c|c|c|}
\hline Lab. No. & County & Detailed Location of Sample & Description of Section \\
\hline 487 & Winnebago & $\underset{1 / 4}{\text { Norway Twp., Sec. } 35 . \mathrm{Nw} .}$ & $\begin{array}{l}5 \mathrm{ft} \text {. brown, fibrous. } \\
2.5 \mathrm{ft} \text {. brown, non-fibrous. }\end{array}$ \\
\hline 488 & Winnebago & $\begin{array}{l}\text { Norway Twp., Sec. } 35 . \\
\text { Same bog as sample No. } \\
487\end{array}$ & $\begin{array}{l}5 \mathrm{ft} \text {. brown, fibrous. } \\
4.5 \mathrm{ft} \text {. brownish black. }\end{array}$ \\
\hline 489 & Worth & $\begin{array}{l}\text { Bristol Twp., Sec. } 6 . \mathrm{Sw} . \\
\text { corner of } \mathrm{Nw} 1 / 4\end{array}$ & $\begin{array}{l}7.5 \mathrm{ft} \text {. brown, fibrous. } \\
3.25 \mathrm{ft} \text { brownish black. }\end{array}$ \\
\hline 490 & Worth & $\begin{array}{clll}\text { Bristol } & \text { Twp., Sec. } & 6 . \\
\text { About } & 150 \text { yds. N. } & \text { of } \\
\text { sample } & \text { No. } 489 & & \end{array}$ & $\begin{array}{l}15.5 \mathrm{ft} \text { brown, fibrous. } \\
\text { darker and finer below. }\end{array}$ \\
\hline 491 & Winnebago & $\begin{array}{l}\text { Center Twp., Sec. } 4, \mathrm{Sw} . \\
\text { corner Nw. 1/4 of } \mathrm{Ne}, 1 / 4\end{array}$ & $\begin{array}{l}3 \mathrm{ft} \text { brown, fibrous. } \\
3 \text { ft. brownish black. }\end{array}$ \\
\hline 492 & Worth & Silver Lake Twp., Sec. 10 & $\begin{array}{l}\text { Upper portion sample No. } \\
486 \text {. }\end{array}$ \\
\hline 493 & Worth & $\begin{array}{l}\text { Silver Lake Twp., Sec. } 22 . \\
600 \text { yds. W. of } \mathrm{E} \text {. line } \\
\text { and } 150 \text { yds. Nw. of } \\
\text { bridge }\end{array}$ & $\begin{array}{l}3 \text { ft. brown, fibrous. } \\
2 \mathrm{ft} \text { brownish black. } \\
6 \mathrm{ft} \text {. brown, lighter below. }\end{array}$ \\
\hline 494 & Winnebago & $\begin{array}{l}\text { On Twp. line Norway and } \\
\text { Center, Sec. 33, Norway } \\
\text { and Sec. 4, Center Twp. } \\
\text { At center of neck }\end{array}$ & $\begin{array}{l}5 \mathrm{ft} \text {. brown, fibrous. } \\
10 \mathrm{ft} \text {. brown, non-fibrous. } \\
6 \mathrm{ft} \text {. reddish brown. }\end{array}$ \\
\hline 495 & Worth & $\begin{array}{l}\text { Silver Lake Twp., Sec. } 10 \text {. } \\
\text { About } 250 \text { yds. Se. of } \\
\text { No. } 486\end{array}$ & $\begin{array}{l}5.5 \text { ft. brownish black. } \\
4 \text { ft. grayish brown. } \\
3 \text { ft. reddish brown. } \\
7 \text { ft. grayish brown, marly. } \\
\text { Bottom not reached. }\end{array}$ \\
\hline 496 & Worth & $\begin{array}{l}\text { Silver Lake Twp.. Sec. } 22 . \\
250 \text { yds. Nw. of No. } 493\end{array}$ & $\begin{array}{l}4 \text { ft. brown, fibrous. } \\
1.5 \text { ft. brownish black. } \\
3.5 \text { ft. light brown. }\end{array}$ \\
\hline 497 & Winnebago & $\begin{array}{l}\text { Norway Twp., on line be- } \\
\text { twcen Secs. } 22 \text { and } 23 \text {. } \\
100 \text { yds. from S. edge } \\
\text { of bog }\end{array}$ & $\begin{array}{l}3 \mathrm{ft} \text {. brown, fibrous. } \\
3.5 \mathrm{ft} \text {. brownish black. } \\
4 \mathrm{ft} \text { light brown. }\end{array}$ \\
\hline 498 & Winnebago & $\begin{array}{l}\text { Center Twp., Sec. } 29.150 \\
\text { yds. from } \\
\text { Nw. } 1 / 4\end{array}$ & $\begin{array}{l}5 \text { ft. brown, fibrous. } \\
2 \text { ft. brownish black. } \\
2 \text { ft. brown, non-fibrous. }\end{array}$ \\
\hline 499 & Winnebago & $\begin{array}{l}\text { Center Twp., on line Secs. } \\
10 \text { and } 11 . \quad 300 \text { yds. } \mathbf{S} \text {. } \\
\text { of road }\end{array}$ & $\begin{array}{l}9 \text { ft. brown, fibrous. } \\
2 \text { ft. brownish black. }\end{array}$ \\
\hline 500 & Winnebago & $\begin{array}{l}\text { Center Twp., Sec. } 4.150 \\
\text { yds. Nw. of sample No. } \\
491\end{array}$ & $\begin{array}{l}3 \text { ft. brown, fibrous. } \\
6 \text { ft. brownish black. } \\
7 \text { ft. reddish brown. }\end{array}$ \\
\hline
\end{tabular}


TABLE II-CONTINUED

\begin{tabular}{|c|c|c|c|}
\hline $\operatorname{Lab}$. No. & County & Detailed Location of Sample & Description of Section \\
\hline 501 & Winnebago & $\begin{array}{l}\text { Center Twp., Sec. 10. } 125 \\
\text { yds. E. of road and } 100 \\
\text { yds. from ditch }\end{array}$ & $\begin{array}{l}9 \text { ft. brown, fibrous. } \\
4.5 \text { ft. brownish black. }\end{array}$ \\
\hline 502 & Winnebago & $\begin{array}{l}\text { Center Twp., Sec. } 29.150 \\
\text { yds. due east from sam- } \\
\text { ple No. } 498\end{array}$ & $\begin{array}{l}5 \text { ft. brown, fibrous. } \\
4 \text { ft. brownisb black. }\end{array}$ \\
\hline 503 & Winnebago & $\begin{array}{l}\text { Norway Twp, on line be- } \\
\text { tween Secs. } 17 \text { and } 18 \text {. } \\
\text { Middle of bog }\end{array}$ & $\begin{array}{l}5 \text { ft. brown, fibrous. } \\
2 \text { ft. brownish black. }\end{array}$ \\
\hline 504 & Winnebago & $\begin{array}{c}\text { Center Twp., Sec. 14. Cen- } \\
\text { ter Nw. } 1 / 4 \text { of } N w .1 / 4\end{array}$ & $\begin{array}{l}9 \text { ft. brown, fibrous. } \\
6 \text { ft. dark brown. } \\
9 \text { ft. reddish brown. } \\
\text { Bottom not reached. }\end{array}$ \\
\hline 505 & Winnebago & $\begin{array}{l}\text { Norway Twp., Sec. } 35 . \\
\text { Center Nw. } 1 / 4\end{array}$ & $\begin{array}{l}5 \text { ft. brown, fibrous. } \\
4 \text { ft. brown, non-fibrous. } \\
8 \text { ft. brownish blacls to red- } \\
\text { dish below. } \\
6 \text { ft. grayish brown, marly. }\end{array}$ \\
\hline 506 & Winnebago & $\begin{array}{l}\text { Norway Twp., Sec. } 35 . \\
\text { Sw. } 1 / 4 . \text { E. of sample } \\
\text { No. } 488 \text { and } 150 \text { yds. } \\
\text { from east edge }\end{array}$ & $\begin{array}{l}6.5 \mathrm{ft} \text {. brown, fibrous. } \\
1 \mathrm{ft} \text {. brownish black. }\end{array}$ \\
\hline 507 & Winnebago & $\begin{array}{l}\text { Norway Twp., on line be- } \\
\text { tween Secs, } 22 \text { and } 23 . \\
150 \text { yds. from north } \\
\text { side of sections }\end{array}$ & $\begin{array}{l}5 \text { ft. brown, fibrous. } \\
4 \text { ft. brownish black. } \\
3.5 \text { ft. reddish brown. }\end{array}$ \\
\hline 508 & Winnebago & $\begin{array}{l}\text { Norway Twp., on road be- } \\
\text { tween Secs. } 14 \text { and } 15 . \\
100 \text { yds. N. of middle } \\
\text { line }\end{array}$ & $\begin{array}{l}5 \text { ft. brown, fibrous. } \\
4 \text { ft. brownish black. } \\
4 \text { ft. brown, non-fibrous. } \\
2 \text { ft. reddish brown. } \\
5 \text { ft. grayish, peaty marl. }\end{array}$ \\
\hline $\begin{array}{r}509 \\
(\mathrm{Top})\end{array}$ & Winnebago & $\begin{array}{l}\text { Norway } \text { Twp., on line } \\
\text { Secs. } 7 \text { and } 8 . \text { Middle } \\
\text { of bog }\end{array}$ & $\begin{array}{l}3 \text { ft. brown, fibrous. } \\
2 \text { ft. brownish black. } \\
4 \text { ft. grayish brown, marly }\end{array}$ \\
\hline 510 & Winnebago & $\begin{array}{c}\text { Norway Twp. Same as } \\
\text { sample No. } 509\end{array}$ & $\begin{array}{l}\text { Same as No. 509, lower } \\
\text { part of section. }\end{array}$ \\
\hline 689 & Hancock & $\begin{array}{l}\text { Madison } T w p ., \text { Sec. } 16 . \text { Se. } \\
1 / 4 \text { of } \mathrm{Sw} .1 / 4 \text { near } \mathrm{Nw} . \\
\text { corner }\end{array}$ & 4 ft. brown, fibrous. \\
\hline 690 & Hancock & $\begin{array}{l}\text { Madison Twp., Sec. } 20 . \\
\text { Near Ne. corner }\end{array}$ & $3.5 \mathrm{ft}$. brown, fibrous. \\
\hline 691 & Webster & 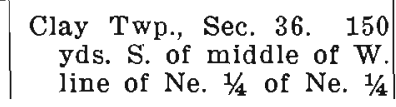 & 3 ft. brown, fibrous. \\
\hline
\end{tabular}


TABLE II-CONTINUED

\begin{tabular}{|c|c|c|c|}
\hline Lab. No. & County & Detailed Location of Sample & Description of Section \\
\hline 692 & Webster & $\begin{array}{l}\text { Burnside Twp., Sec. } 31 . \\
200 \text { yds. Sw. of center } \\
\text { of section }\end{array}$ & 2 ft. brown, fibrous. \\
\hline 693 & Webster & $\begin{array}{l}\text { Lost Grove Twp., on sec } \\
\text { tion line Secs. } 13 \text { and } \\
\text { 24, near middle }\end{array}$ & 3 ft. brown, fibrous. \\
\hline 557 & Winnebago & $\begin{array}{l}\text { Mt. Valley Twp., Sec. } 6 \\
\text { Middle of W. side of Ne } \\
1 / 4 \text { of Se. } 1 / 4\end{array}$ & $\begin{array}{l}8 \mathrm{ft} \text {. brown, fibrous. } \\
\text { Gray, marly peat below. }\end{array}$ \\
\hline 558 & Winnebago & $\begin{array}{l}\text { Mt. Valley Twp., Sec. } 6 . \\
\text { Near Ne. corner }\end{array}$ & $\begin{array}{l}8 \text { ft. brown, fibrous. } \\
1 \text { ft. brown. }\end{array}$ \\
\hline 559 & Winnebago & $\begin{array}{l}\text { Mt. Valley Twp., Sec. } 22 . \\
100 \text { yds. Sw. from Ne. } \\
\text { corner Nw. } 1 / 4 \text { of Nw. } 1 / 4\end{array}$ & $\begin{array}{l}9 \mathrm{ft} \text {. brown, fibrous. } \\
1 \mathrm{ft} \text {. brownish black. }\end{array}$ \\
\hline 560 & Winnebago & $\begin{array}{l}\text { Mt. Valley Twp., Sec. } 22 . \\
\text { Center Ne. } 1 / 4 \text { of Ne. } 1 / 4\end{array}$ & $\begin{array}{l}9 \text { ft. brown, fibrous. } \\
1 \text { ft. brown. }\end{array}$ \\
\hline 561 & Winnebago & $\begin{array}{l}\text { Mt. Valley Twp., Sec. } 17 . \\
\text { Center Nw. } 1 / 4 \text { of Nw. } 1 / 4\end{array}$ & $\begin{array}{l}7 \text { ft. brown, fibrous. } \\
6 \text { ft. brown. }\end{array}$ \\
\hline 562 & Winnebago & $\begin{array}{l}\text { Mt. Valley Twp., Sec. } 17 . \\
\text { Middle N. line of } \mathrm{Sw} . \\
1 / 4 \text { of } \mathrm{Nw} .1 / 4\end{array}$ & $\begin{array}{l}9 \mathrm{ft} \text { brown, fibrous. } \\
6 \mathrm{ft} \text {. brown. } \\
\text { Clayey, sandy peat below. }\end{array}$ \\
\hline 563 & Winnebago & $\begin{array}{l}\text { Mt. Valley Twp., Sec. } 17 . \\
\text { Se. corner Sw. } 1 / 4 \text { of } \mathrm{Nw} \text {. } \\
1 / 4\end{array}$ & $\begin{array}{l}8 \mathrm{ft} \text { brown, fibrous. } \\
\text { Peat continued down } 25 \mathrm{ft} \text {. } \\
\text { but too soft to sample. }\end{array}$ \\
\hline 564 & Winnebago & $\begin{array}{l}\text { Mt. Valley Twp., Sec. } 17 . \\
\text { Center Ne. } 1 / 4 \text { of } \mathrm{Nw} .1 / 4,\end{array}$ & $\begin{array}{l}9 \text { ft. brown, fibrous. } \\
9 \mathrm{ft} \text { brown, non-fibrous. } \\
\text { Peat continues down more } \\
\text { than } 30 \mathrm{ft} \text {. }\end{array}$ \\
\hline 565 & Winnebago & $\begin{array}{l}\text { Mt. Valley Twp., Sec. } 8 . \\
\text { Center Se. } 1 / 4 \text { of Se. } 1 / 4\end{array}$ & $\begin{array}{l}7 \text { ft. brown, fibrous. } \\
3 \text { ft. brownish black. } \\
\text { Darker below. }\end{array}$ \\
\hline 566 & Winnebago & $\begin{array}{l}\text { Mt, Valley Twp., Sec. } 8 . \\
100 \text { yds. Nw. of Se. cor- } \\
\text { ner of Sw. } 1 / 4 \text { of Se. } 1 / 4\end{array}$ & $\begin{array}{l}5 \text { ft. brown, fibrous. } \\
4 \text { ft. brownish black. }\end{array}$ \\
\hline 567 & Winnebago & $\begin{array}{l}\text { Mt. Valley Twp., Sec. } 16 . \\
\text { Center Se. } 1 / 4 \text { of Sw. } 1 / 4\end{array}$ & 8 ft. brown, fibrous. \\
\hline 568 & Winnebago & $\begin{array}{l}\text { Mt: Valley Twp., Sec. } 16 \\
100 \text { yds. Nw. from Se. } \\
\text { corner of } \mathrm{Nw} .1 / 4 \text { of } \mathrm{Sw} . \\
1 / 4\end{array}$ & 7 ft. brown, fibrous. \\
\hline
\end{tabular}


TABLE II-CONTINUED

\begin{tabular}{|c|c|c|c|}
\hline Lab.No. & County & Detailed Location of Sample & Description of Section \\
\hline$(069)$ & Winnebago & $\begin{array}{l}\text { Mt. Valley Twp., Sec. } 16 . \\
150 \text { yds. Sw. from sam- } \\
\text { ple No. } 568\end{array}$ & 7 ft. brown, fibrous. \\
\hline 569 & Winnebago & $\begin{array}{l}\text { Mt. Valley Twp., Sec. } 17 . \\
\text { Center Se. 1/4 of Sw. 1/4 }\end{array}$ & 7 ft. brown, fibrous. \\
\hline 570 & Winnebago & $\begin{array}{l}\text { Mt. Valley Twp., Sec. } 17 . \\
\text { Center Nw. 1/4 of Se. } 1 / 4\end{array}$ & $\begin{array}{l}7 \text { ft. brown, fibrous } \\
6 \text { ft. brown, non-fibrous. }\end{array}$ \\
\hline 571 & Winnebago & $\begin{array}{l}\text { Mt. Valley Twp., Sec. } 17 . \\
200 \text { yds. N. Sec. line }\end{array}$ & $7 \mathrm{ft}$. brown, fibrous. \\
\hline 573 & Winnebago & $\begin{array}{l}\text { Mt. Valley Twp., Sec. } 17 . \\
\text { Center Sw. } 1 / 4 \text { of Se. } 1 / 4\end{array}$ & $\begin{array}{l}13 \text { ft. brown, fibrous, be } \\
\text { coming less fibrous below. }\end{array}$ \\
\hline 572 & Hancock & $\begin{array}{cccc}\text { Garfield } & \text { Twp., } & \text { Sec. } & 10 \\
\text { Center Ne. } 1 / 4 & \text { of } & \text { Se. } & 1 / 4\end{array}$ & $\begin{array}{l}5 \text { ft. brown, fibrous. } \\
1 \text { ft. brownish black. }\end{array}$ \\
\hline 574 & Hancock & $\begin{array}{l}\text { Concord Twp., Sec. 5. } 100 \\
\text { yds. Sw. from Ne. cor- } \\
\text { ner of Nw. } 1 / 4 \text { of Ne. } 1 / 4\end{array}$ & $\begin{array}{l}8 \text { ft. brown, fibrous. } \\
1 \text { ft. brown, non-fibrous. }\end{array}$ \\
\hline 590 & Cerro Gordo & $\begin{array}{l}\text { Grant Twp., line between } \\
\text { Secs. } 34 \text { and } 35 \text {, near } \\
\text { middle }\end{array}$ & 9 ft. brown, fibrous. \\
\hline 595 & Cerro Gordo' & $\begin{array}{lll}\text { Grant } & \text { Twp., Sec. } 25 . & 200 \\
\text { yds. south sample } & \text { No. } \\
590 & & \end{array}$ & 5 ft. brown, fibrous. \\
\hline 605 & Cerro Gordo & $\begin{array}{l}\text { Grant Twp., Sec. } 25.3^{3} 00 \\
\text { yds. E. of center of sec- } \\
\text { tion }\end{array}$ & $\begin{array}{l}5 \text { ft. brown, fibrous. } \\
4 \text { ft. brown, non-fibious. } \\
5 \text { ft. reddish brown. }\end{array}$ \\
\hline 606 & Cerro Gordo & $\begin{array}{l}\text { Grant Twp., Sec. } 25 . \quad 150 \\
\text { yds. E. from center of } \\
\text { section }\end{array}$ & $\begin{array}{l}5 \text { ft. brown, fibrous. } \\
3 \text { ft. brown, non-fibrous. } \\
4 \text { ft. marly peat. }\end{array}$ \\
\hline 607 & Cerro Gordo & $\begin{array}{l}\text { Grant Twp., Sec. } 25.400 \\
\text { yds. S. and } 200 \text { yds. W. } \\
\text { of Ne. corner of Nw. } 1 / 4\end{array}$ & $\begin{array}{l}4 \text { ft. brown, fibrous. } \\
4 \text { ft. brownish black. }\end{array}$ \\
\hline 608 & Cerro Gordo. & $\begin{array}{l}\text { Grant Twp., Sec. } 15 . \quad 200 \\
\text { yds. S. and } 200 \text { yds. W. } \\
\text { of Ne. corner of section }\end{array}$ & $\begin{array}{l}5 \text { ft. brown, fibrous. } \\
3 \text { ft. brown, non-fibrous. } \\
2 \text { ft. reddish brown. }\end{array}$ \\
\hline 609 & Cerro Gordo & $\begin{array}{l}\text { Grant Twp., Sec. } 15 . \\
\text { yds. W. of sample No. } \\
608\end{array}$ & 4 ft. brown, fibrous. \\
\hline 610 & Cerro Gordo & $\begin{array}{l}\text { Grant Twp. Sec. } 22 . \text { Cen- } \\
\text { ter Nw. 1/4 of Sw. 1/4 }\end{array}$ & $\begin{array}{l}7 \mathrm{ft} \text {. brown, fibrous. } \\
3 \mathrm{ft} \text {. brown, non-fibrous. }\end{array}$ \\
\hline
\end{tabular}


TABLE II-CONTINUED

\begin{tabular}{|c|c|c|c|}
\hline Lab.No. & County & Detailed Location of Sample & Description of Section \\
\hline 611 & Cerro Gordo & $\begin{array}{l}\text { Grant Twp., Sec. } 22.250 \\
\text { yds. S. from sample No. } \\
610\end{array}$ & $\begin{array}{l}5 \mathrm{ft} \text {. brown, fibrous. } \\
1 \mathrm{ft} \text {. brown, non-fibrous. }\end{array}$ \\
\hline 613 & Cerro Gordo & $\begin{array}{l}\text { Grant Twp., Sec. } 31.200 \\
\text { yds. E. from center of } \\
\text { section }\end{array}$ & $\begin{array}{l}5 \mathrm{ft} \text {. brown, fibrous. } \\
3 \mathrm{ft} \text { brown, non-fibrous. }\end{array}$ \\
\hline 627 & Cerro Gordo & $\begin{array}{l}\text { Union Twp., Sec. } 20 . \quad 200 \\
\text { yds. S. of Ne. corner of } \\
\text { Se. } 1 / 4\end{array}$ & $6 \mathrm{ft}$. brown, fibrous. \\
\hline 628 & Cerro Gordo & $\begin{array}{l}\text { Clear Lake Twp., Sec. } 26 . \\
400 \text { yds. N. of center of } \\
\text { Se. } 1 / 4\end{array}$ & $\begin{array}{l}5 \mathrm{ft} . \text { brown, fibrous. } \\
4 \mathrm{ft} \text { brown, non-fibrous. }\end{array}$ \\
\hline 629 & Emmet & $\begin{array}{l}\text { Iowa Lake Twp., Sec. } 34 . \\
\text { Center Nw. } 1 / 4 \text { of Se. } 1 / 4 \\
\end{array}$ & 3 ft. brown, fibrous. \\
\hline 630 & Emmet & $\begin{array}{l}\text { Iowa Lake Twp., Sec. } 34 . \\
\text { Center Sw. } 1 / 4 \text { of Ne. } 1 / 4 \\
\end{array}$ & 5 ft. brown, fibrous. \\
\hline 631 & Emmet & $\begin{array}{l}\text { Iowa Lake Twp., Sec. } 34 \text {. } \\
\text { Center of Sw. } 1 / 4\end{array}$ & 5 ft. brown, fibrous. \\
\hline 632 & Emmet & $\begin{array}{l}\text { Iowa Lake Twp., Sec. } 34 . \\
\text { Center Nw. 1/4 of Nw. } 1 / 4\end{array}$ & 4 ft. brown, fibrous. \\
\hline 633 & Kossuth.. & $\begin{array}{l}\text { Swea Twp., Sec. 4. Center } \\
\text { Nw. } 1 / 4 \text { of Ne. } 1 / 4\end{array}$ & 4 ft. brown, fibrous. \\
\hline 642 & Wright & $\begin{array}{l}\text { Lake Twp., Sec. } 1.500 \\
125 \text { yds. from N. side of } \\
\text { bog }\end{array}$ & $\begin{array}{l}4 \mathrm{ft} \text { brown, flbrous. } \\
\text {-Thin sand seam below. }\end{array}$ \\
\hline 643 & Wright & $\begin{array}{l}\text { Lake Twp., Sec. } 1 . \quad 125 \\
\text { yds. from S. edge of bog } \\
\text { along ditch }\end{array}$ & 4 ft. brown, fibrous. \\
\hline 644 & Hancock & $\begin{array}{l}\text { German Twp., Sec. 4. Sw. } \\
1 / 4 \text {, on N. line } 100 \mathrm{yds} \text {. } \\
\text { W. of ditch }\end{array}$ & 4 ft. brown, fibrous. \\
\hline 645 & Hancock & 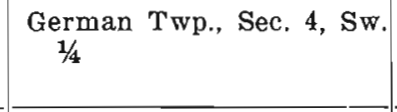 & $\begin{array}{l}\text { Same as No. } 644 \text {, taken be- } \\
\text { low } 4 \mathrm{ft} \text {. down to } 6.5 \text {. } \\
\text { Brownish black peat. }\end{array}$ \\
\hline 646 & Hancock & $\begin{array}{l}\text { German Twp., Sec. 9. } 200 \\
\text { yds. S. of Sec. line on } \\
\text { ditch }\end{array}$ & 2.5 ft. brown, fibrous. \\
\hline
\end{tabular}


PEAT DEPOSITS IN IOW'A

TABLE II-ConTINUED

\begin{tabular}{|c|c|c|c|}
\hline Lab.No. & County & Detailed Location of Sample & Description of Section \\
\hline 647 & $\begin{array}{c}\text { Hancock } \\
.\end{array}$ & $\begin{array}{l}\text { Crystal Twp., Sec. } 23 \text {. On } \\
\text { N. and S. } 1 / 2 \text { Sec. line, } \\
150 \text { yds. from S. side of } \\
\text { bog }\end{array}$ & $3.5 \mathrm{ft}$. brown, fibrous. \\
\hline 648 & Hancock & $\begin{array}{l}\text { Madison Twp., Sec. } 19 . \\
200 \text { yds. east of middle, } \\
\text { S. line of } \mathrm{Sw} .1 / 4 \text { of } \mathrm{Nw} \text {. } \\
1 / 4\end{array}$ & 4 ft. brown, fibrous. \\
\hline 649 & Webster & $\begin{array}{l}\text { Clay Twp., Sec. } 36 \text {. On } \\
\text { N. Iine } 250 \text { yds. W. of } \\
\text { the middle }\end{array}$ & 4 ft. brown, fibrous. \\
\hline 650 & Webster & $\begin{array}{l}\text { Burnside Twp., Sec. } 31 . \\
\text { Near middle south line }\end{array}$ & 2 ft. brown, fibrous. \\
\hline 685 & Wright & $\begin{array}{l}\text { Belmond Twp., Sec. } 17 . \\
\text { On east line, } 300 \text { yds. } \mathrm{S} \text {. } \\
\text { of corner }\end{array}$ & $5 \mathrm{ft}$. brown, fibrous. \\
\hline 686 & Hancock & $\begin{array}{llll}\text { Crystal } & \text { Twp., } & \text { Sec. } & 35 . \\
\text { Center Ne. } 1 / 4 & \end{array}$ & $4 \mathrm{ft}$. brown, fibrous. \\
\hline 687 & Hancock & $\begin{array}{l}\text { Crystal Twp., Sec. } 25 . \\
200 \text { yds. NNe. from Sw. } \\
\text { corner }\end{array}$ & 3.5 et. brown, fibrous. \\
\hline 688 & Hancock & $\begin{array}{l}\text { Crystal Twp., Sec. } 25.250 \\
\text { yds. S. of Nw. corner }\end{array}$ & $4 \mathrm{ft}$. brown, fibrous. \\
\hline
\end{tabular}




\section{TABLE III}

Gives laboratory number, proximate chemical analyses of air dried samples, and calorimetric analyses of Iowa peats.

\begin{tabular}{|c|c|c|c|c|c|c|}
\hline & \multirow[b]{2}{*}{$\begin{array}{l}\text { Laboratory } \\
\text { Number }\end{array}$} & \multicolumn{4}{|c|}{ Proximate Analysis } & \multirow{2}{*}{$\begin{array}{l}\text { Calorime- } \\
\text { tric Anal- } \\
\text { ysis in } \\
B . T . \text { Os. }\end{array}$} \\
\hline & & Moisture & $\begin{array}{c}\text { Volatile } \\
\text { and Com- } \\
\text { bustible } \\
\text { Matter }\end{array}$ & $\begin{array}{l}\text { Fixed } \\
\text { Carbon }\end{array}$ & $A s h$ & \\
\hline 350 & 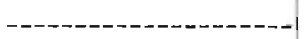 & 6.33 & 44.16 & 8.26 & 41.25 & 5535 \\
\hline 351 & 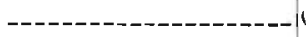 & 7.49 & 47.53 & 11.52 & 33.46 & 6815 \\
\hline 352 & 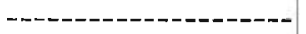 & 6.61 & 40.59 & 5.66 & 47.14 & 4878 \\
\hline 353 & 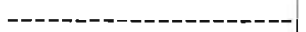 & 7.13 & 49.31 & 11.13 & 32.43 & 6234 \\
\hline 354 & ---- & $8.66^{\circ}$ & 52.47 & 15.38 & 23.49 & 7699 \\
\hline 355 & 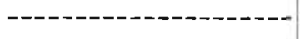 & 5.05 & 32.10 & 5.41 & 57.44 & $--\cdots-1--\overline{1}$ \\
\hline $\begin{array}{l}356 \\
357\end{array}$ & 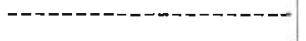 & $\begin{array}{l}4.40 \\
4.31\end{array}$ & $\begin{array}{l}42.39 \\
30.83\end{array}$ & 6.65 & 46 & 4691 \\
\hline $\begin{array}{l}357 \\
358\end{array}$ & 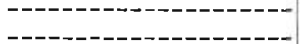 & $\begin{array}{l}4.31 \\
5.36\end{array}$ & $\begin{array}{l}50.85 \\
24.21\end{array}$ & $\begin{array}{l}5.05 \\
5.88\end{array}$ & $\begin{array}{l}59.83 \\
64.55\end{array}$ & $-1-1-$ \\
\hline 359 & 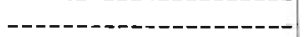 & 4.87 & 39.85 & 6.35 & 48.93 & $497 \overline{3}$ \\
\hline 360 & 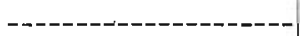 & 5.67 & 47.67 & 6.03 & 40.63 & 4894 \\
\hline 361 & 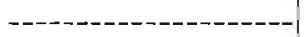 & 4.84 & 29.42 & 4.83 & 60.91 & \\
\hline 362 & 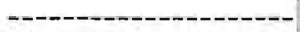 & 5.48 & 31.90 & 7.77 & 54.85 & 4317 \\
\hline 363 & - & 6.60 & 40.86 & 4.34 & 48.20 & 5626 \\
\hline 364 & 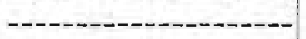 & 3.99 & 50.45 & 5.31 & 40.25 & 4863 \\
\hline 365 & 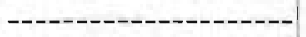 & 5.63 & 53.55 & 8.31 & 32.51 & 6047 \\
\hline 366 & - - - - - - - - & 2.03 & 10.33 & .18 & 87.46 & 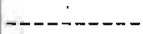 \\
\hline 367 & 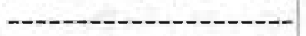 & 3.76 & 20.27 & 3.51 & 72.46 & $\mid---$ \\
\hline 368 & - & 4.49 & 39.00 & 4.42 & 52.09 & ------ - \\
\hline 369 & 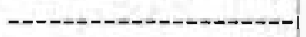 & 3.80 & 35.84 & 2. & 57.61 & \\
\hline 370 & 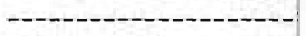 & 3.91 & 34.99 & 3.57 & 57.53 & --------- \\
\hline 371 & - - - - - & 3.64 & 32.90 & 1. & 61.96 & $\infty--\infty-\infty$ \\
\hline 372 & 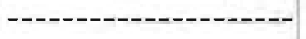 & 2.91 & 19.21 & 1.9 & 75.89 & ------- \\
\hline 373 & 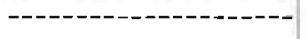 & 5.80 & 33.83 & 5. & 55.21 & 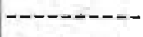 \\
\hline 374 & ------- & $\begin{array}{l}5.82 \\
3.28\end{array}$ & 28.94 & 6.43 & 58.81 & --------- \\
\hline 375 & 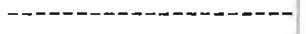 & 3.28 & 15.04 & .07 & 81.61 & $----\infty-$ \\
\hline 376 & 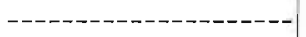 & 3.08 & 26.54 & 1.61 & 68.77 & |------- \\
\hline 377 & 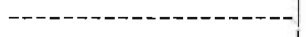 & 4.12 & 35.41 & 2.97 & 57.50 & $---\infty$ \\
\hline 378 & --- & 2.94 & 11. & .80 & 84 & - \\
\hline 379 & ----- & 3.07 & 17. & 2 . & 77.41 & --------- \\
\hline 380 & 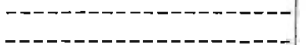 & $\begin{array}{l}1.86 \\
2.27\end{array}$ & $\begin{array}{l}13.90 \\
12.21\end{array}$ & .9 & 83.29 & ------- \\
\hline 381 & 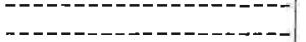 & $\begin{array}{l}2.27 \\
3.43\end{array}$ & 8.6 & .03 & 87.89 & $-\cdots$ \\
\hline 383 & $-\ldots$ & 3.37 & 11.80 & .54 & 84.29 & $x-2-2-1$ \\
\hline 383. & 5 - & 4.47 & 17.40 & 1.87 & 76.26 & n---- \\
\hline 384 & ------ & 3.66 & 14.99 & 2.75 & 78.60 & $\cdots----$ \\
\hline 385 & ----------- & 4.00 & 23.92 & 1.85 & 70.23 & - \\
\hline 386 & 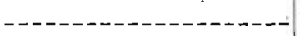 & 4.07 & 12.05 & .94 & 82.94 & $-\ldots$ \\
\hline 387 & 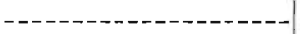 & 2.85 & 12.50 & .56 & 84.09 & - \\
\hline 388 & --- - - - - - & 2.58 & 9.47 & .00 & 87.95 & -.--_-_- \\
\hline 389 & 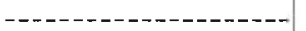 & 2.59 & 19.89 & .00 & 77.52 & ------- \\
\hline 390 & 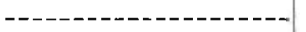 & 1.86 & 11.90 & .32 & 85.92 & - - - - - \\
\hline 391 & 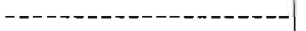 & 2.60 & 13.27 & .35 & 83.78 & -- - \\
\hline 392 & 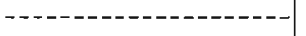 & 2.71 & 15.08 & .86 & 81.35 & $-\ldots \ldots-n$ \\
\hline 393 & 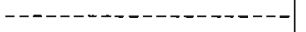 & 3.28 & 18.10 & 1.00 & 77.62 & $---\infty-n$ \\
\hline
\end{tabular}


TABLE. 1II-ContinueD

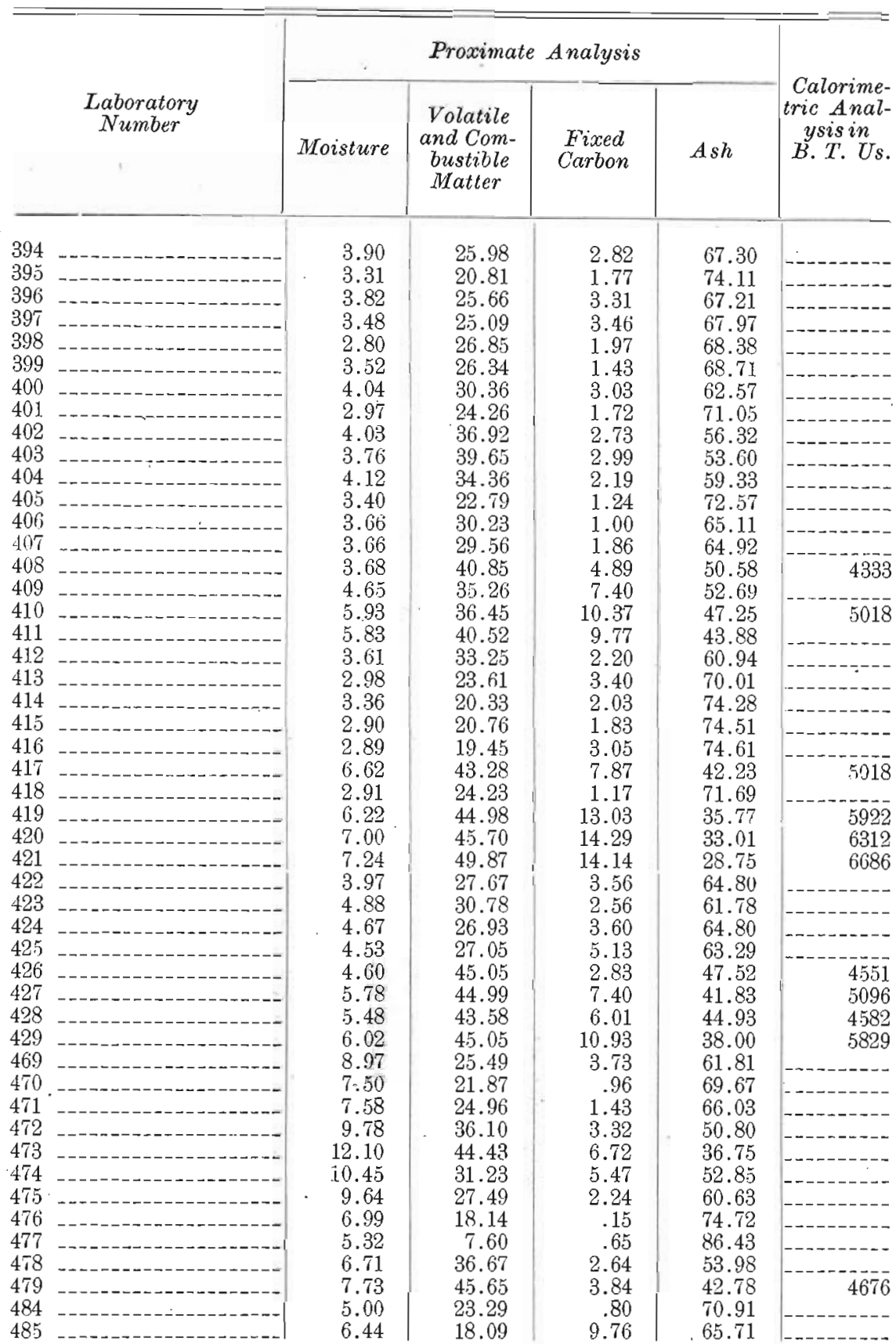


ANALYSES OF PEATS

TABLE III-Continued

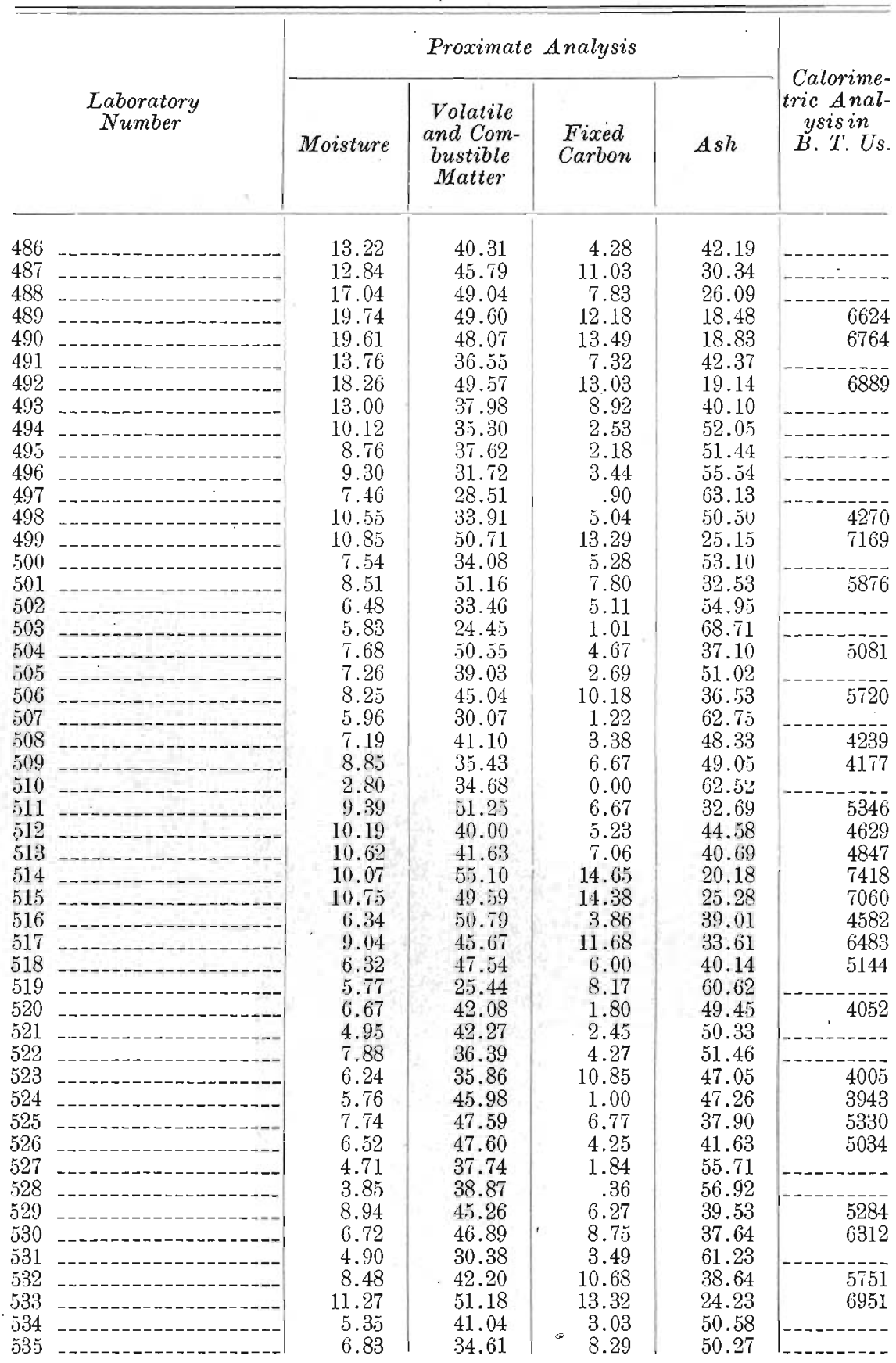


TABLE III-CONTINUED

\begin{tabular}{|c|c|c|c|c|c|c|}
\hline & \multirow[b]{2}{*}{$\begin{array}{c}\text { Laboratory } \\
\text { Number }\end{array}$} & \multicolumn{4}{|c|}{ Proximate Analysis } & \multirow[b]{2}{*}{$\begin{array}{c}\text { Calorime } \\
\text { tric A nal } \\
\text { ysis in } \\
B . T . U s\end{array}$} \\
\hline & & Moisture & $\begin{array}{l}\text { Volatile } \\
\text { and Com- } \\
\text { bustible } \\
M a\end{array}$ & $\begin{array}{l}\text { Fixed } \\
\text { Carbon }\end{array}$ & $A s h$ & \\
\hline 536 & 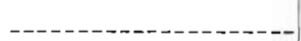 & 8.94 & 49.02 & 13.33 & 28.71 & \\
\hline 537 & 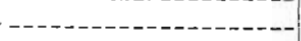 & 8.90 & 46.36 & 10.42 & 34.32 & 6078 \\
\hline 538 & 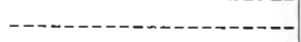 & 4.39 & 48.45 & 3.24 & 43.92 & 4442 \\
\hline 539 & ----- & ¿3.91 & 44.41 & 12.44 & 29.24 & 6203 \\
\hline 540 & -non & 8.04 & 39.03 & 6.70 & 46.23 & 4691 \\
\hline 541 & 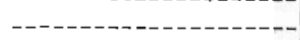 & 6.16 & 46.07 & 2.10 & 45.67 & 4052 \\
\hline 542 & - - - - - - - - - & 6.71 & 41.95 & 8.13 & 43.21 & 5159 \\
\hline 543 & 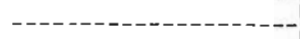 & 5.38 & 39.06 & 7.11 & 48.45 & 4800 \\
\hline 544 & 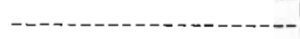 & 3.44 & 30.11 & 1.68 & 64.77 & \\
\hline 545 & - - & 6.73 & 46.90 & 18.22 & 28.15 & 6483 \\
\hline 546 & 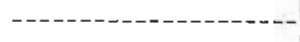 & 5.56 & 38.00 & 5.47 & 50.97 & ---- \\
\hline 547 & --- & 3.62 & 40.88 & 1.08 & 54.42 & . \\
\hline 548 & - & 5.83 & 39.90 & .03 & 54.24 & 列 \\
\hline $\begin{array}{l}549 \\
550\end{array}$ & - & $\begin{array}{l}4.40 \\
3.30\end{array}$ & $\begin{array}{l}29.65 \\
37.18\end{array}$ & 4.65 & $\begin{array}{l}61.30 \\
58.17\end{array}$ & ---- \\
\hline 551 & 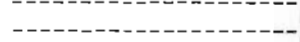 & 6.88 & 46.54 & $\begin{array}{l}1.0 \cdot 0 \\
5.50\end{array}$ & $\begin{array}{l}50.17 \\
41.08\end{array}$ & $495 \overline{6}$ \\
\hline 552 & 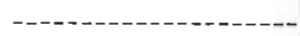 & 8.03 & 51.10 & 11.03 & 29.84 & 6702 \\
\hline 553 & 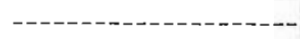 & 9.50 & 48.86 & 10.62 & 31.02 & 5844 \\
\hline 554 & -- - & 5.49 & 53.51 & 4.49 & 36.51 & 5050 \\
\hline 555 & --------- & 4.26 & 27.96 & 4.13 & 63.65 & $---\ldots$ \\
\hline 556 & - -- & 2.35 & 28.01 & 4. & 65.26 & - \\
\hline 557 & 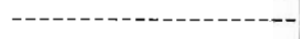 & 6.92 & 52.41 & 5.17 & 35.50 & 5408 \\
\hline 558 & - -------- & 5.16 & 44.60 & 2.88 & 47.36 & 4364 \\
\hline 559 & 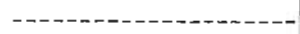 & 7.45 & 47.62 & 13.75 & 31.18 & 6655 \\
\hline 560 & 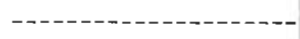 & 4.55 & 49.29 & 6.97 & 39.19 & 5377 \\
\hline 561 & - - & 5.7 & 44 & 10. & 39.34 & 5026 \\
\hline 562 & - - - & 5.7 & 51. & 9. & 33.55 & 6000 \\
\hline 563 & 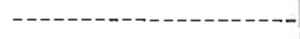 & 5.89 & 63.59 & 16 & 14.13 & 8431 \\
\hline 564 & 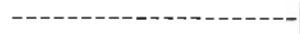 & 5.97 & 49.89 & 6.01 & 38.13 & 4863 \\
\hline 565 & - & 6. & 44.31 & 8.70 & 40.61 & 5455 \\
\hline 566 & 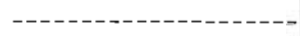 & 4.50 & 42.30 & 12: & 41.00 & 5953 \\
\hline 567 & $-----\ldots-c--------$ & 6 . & 51. & 12. & 29.55 & 6421 \\
\hline 568 & - & 5.29 & 38.83 & 11 & 44.19 & 5564 \\
\hline 569 & 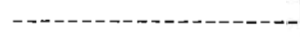 & 6.60 & 49.70 & 11.60 & 32.10 & 6405 \\
\hline 570 & 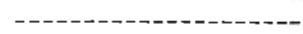 & 5. & 49.82 & 6.37 & 37.82 & 5392 \\
\hline 571 & 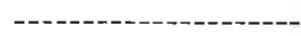 & 6.4 & 50 & 13.87 & 29.40 & 7044 \\
\hline 572 & --n-- - - & 7.4 & 44. & 12 & 35.26 & 6109 \\
\hline 573 & 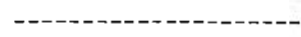 & 7.85 & 53.41 & 3. & 35 & 5253 \\
\hline 574 & 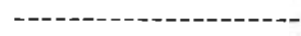 & 8.09 & 51.50 & 13.39 & 27.02 & 7138 \\
\hline 575 & ------- & 7.52 & 55.53 & 3.03 & 33.92 & 4863 \\
\hline 576 & - - & 9.13 & 52.75 & 14.87 & 23.25 & 7247 \\
\hline 577 & 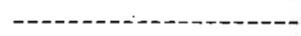 & 6.9 & 46.27 & 9.08 & 37.67 & 5657 \\
\hline 578 & ----- & 3.70 & 39. & & 52.07 & \\
\hline 579 & & 17.65 & 38.42 & 11. & 31.97 & 6499 \\
\hline 580 & - - - - & 5.12 & 40.77 & 5.62 & 48.49 & 4691 \\
\hline 581 & $-----n-\infty$ & 4.71 & 23.96 & 4.34 & 66.99 & \\
\hline 58 & --- & 4.35 & 35.20 & 2.31 & 58.14 & $\cdots$ \\
\hline 583 & - & 8.20 & 45.35 & 14.57 & 31.88 & 6094 \\
\hline 584 & $--\cdot-$ & 6.98 & 46.12 & 6.90 & 39.995 & 5455 \\
\hline
\end{tabular}


ANALYSES OF PEATS

TABLE III-CONTINUED

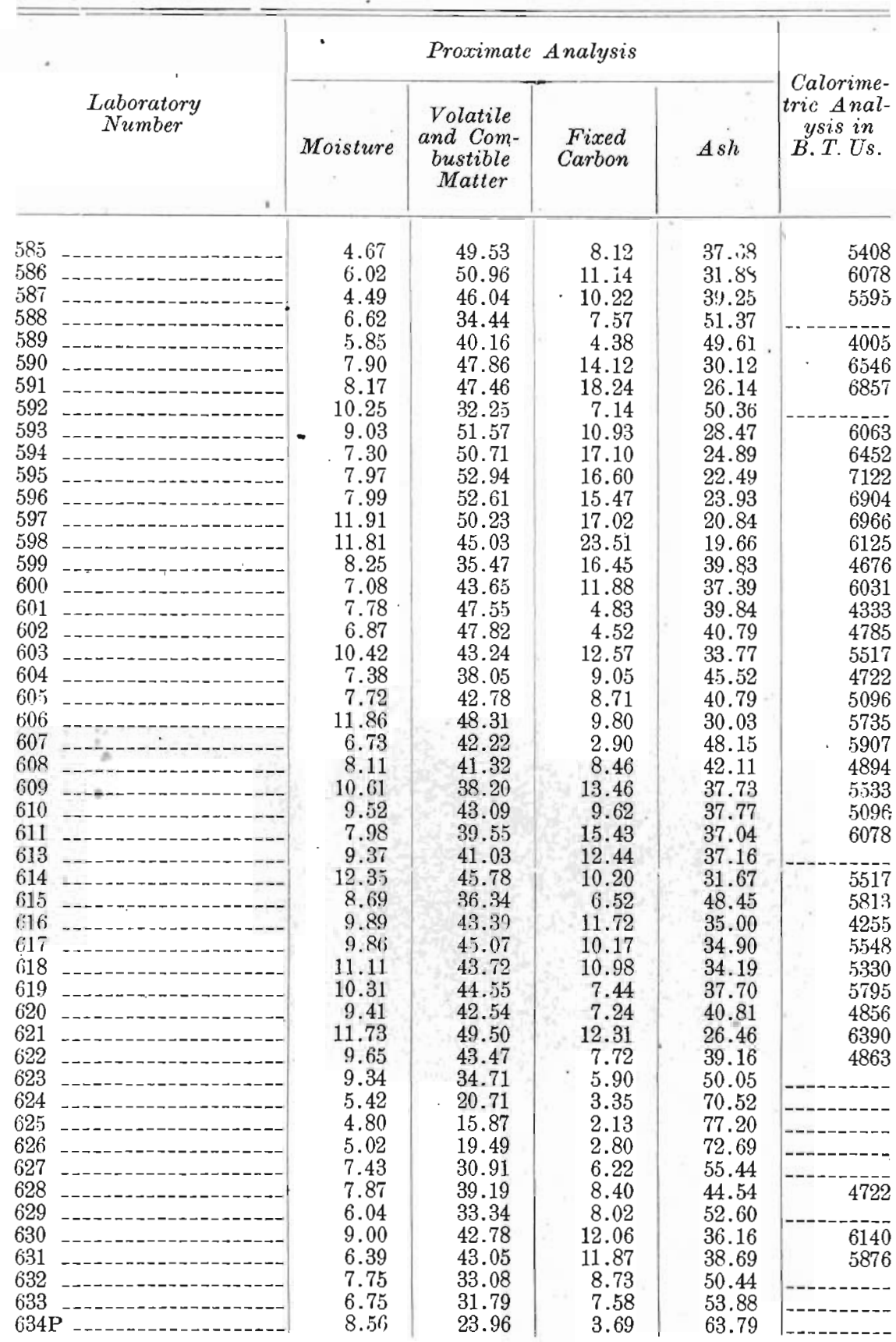


TABIE III-Continued

\begin{tabular}{|c|c|c|c|c|c|c|}
\hline \multirow{2}{*}{\multicolumn{2}{|c|}{$\begin{array}{c}\text { Laboratory } \\
\text { Number }\end{array}$}} & \multicolumn{4}{|c|}{ Proximate Analysis } & \multirow{2}{*}{$\begin{array}{c}{ }^{\circ} \\
\text { Calorime- } \\
\text { tric Anal- } \\
\text { ysis in } \\
\text { B.T.Us. }\end{array}$} \\
\hline & & Moisture & $\begin{array}{c}\text { Volatile } \\
\text { and Com- } \\
\text { bustible } \\
\text { Matter }\end{array}$ & $\begin{array}{l}\text { Fixed } \\
\text { Carbon }\end{array}$ & Ash & \\
\hline $63 ว 5 \mathrm{P}$ & & 11.63 & 40.59 & 21.72 & 26.06 & 6390 \\
\hline $636 \mathrm{P}$ & - & 10.51 & 51.61 & 13.80 & 24.09 & 6811 \\
\hline $637 \mathrm{P}$ & - & 9.58 & 38.03 & 8.24 & 44.15 & 4707 \\
\hline $638 \mathrm{P}$ & - & 10.71 & 44.98 & 8.01 & 36.30 & 5159 \\
\hline $639 \mathrm{P}$ & - & 11.33 & 45.19 & 11.35 & 32.13 & 6031 \\
\hline $640 \mathrm{P}$ & - n & 9.31 & 46.35 & 8.84 & 35.50 & 5096 \\
\hline $641 \mathrm{P}$ & $----\infty-\infty$ & 9.73 & 40.51 & 9.00 & 40.76 & 4894 \\
\hline $642 \mathrm{P}$ & 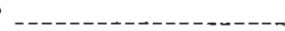 & 12.64 & 43.46 & 14.82 & 29.08 & 6296 \\
\hline $643 \mathrm{P}$ & - & 11.65 & 38.88 & 11.68 & 37.79 & 5986 \\
\hline $644 \mathrm{P}$ & - - & 9.91 & 56.68 & 7.07 & 26.34 & 5798 \\
\hline $645 \mathrm{P}$ & - - & 9.97 & 50.28 & 10.47 & 29.28 & 6099 \\
\hline 638 & - - - - & 6.60 & 40.19 & 9.01 & 44.20 & 5628 \\
\hline $639-$ & 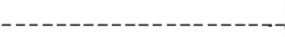 & 8.89 & 45.60 & 8.17 & 37.34 & 5549 \\
\hline 640 - & 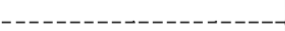 & 7.36 & 41.58 & 7.63 & 43.43 & 4987 \\
\hline $641-$ & 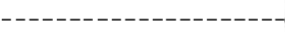 & -5.90 & 40.40 & 10.90 & 42.80 & 6079 \\
\hline 642 & - n- & 6.15 & 49.09 & 17.51 & 27.25 & 5440 \\
\hline $643-$ & - & 5.22 & 46.59 & 15.96 & 32.23 & 6055 \\
\hline 644 & ----n--- & 9.78 & 53.48 & 13.64 & 23.10 & 7169 \\
\hline 645 & $\ldots$ & 6.03 & 34.66 & 5.46 & 53.85 & \\
\hline $646-$ & 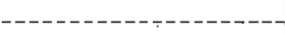 & 6.88 & 49.49 & 13.60 & 30.03 & 6492 \\
\hline 647 - & - - - - & 7.55 & 54.57 & 15.67 & 22.21 & 7278 \\
\hline 648 & 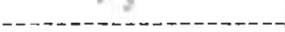 & 7.77 & 49.36 & 15.46 & 27.41 & 7013 \\
\hline 649 & -- & 6.77 & 47.69 & 14.27 & 31.27 & 6468 \\
\hline $650-$ & $-\ldots \ldots-\ldots$ & 7.53 & 40.40 & 11.06 & 41.01 & 5720 \\
\hline $6.51-$ & - - & 5.97 & 21.63 & 1.59 & 70.81 & S------- \\
\hline $652-$ & - & 5.07 & 12.95 & .47 & 81.51 & ---- \\
\hline
\end{tabular}




\section{BIbliography OF IOWA PEAT}

COMPILED BY JAMES H. LEES.

Adair county, ancient peat bed in.

C. A. White, in Geol. Iowa, Vol. I, p. 339. 1870.

Buchanan county, peat in.

S. Calvin, in Ia. Geol. Surv., Vol. VIII, p. 247. 1897.

Beyer, S. W.

Briquetting of peat. Ia. Geol. Surv, Vol. XVIII, p. 28. 1907.

Calvin, $\mathrm{S}$.

Fuel value of peat. Ia. Geol. Surv., Vol. XVII, p. 3. 1906.

Peat in Buchanan county. Ibid., Vol, VIII, p. 247. 1897.

Peat in Cerro Gordo county. Ibid., Vol. VII, p. 192.1896.

Peat in Chickasaw county. Ibid, Vol, XIII, p. 289. 1902.

Peat in Pleistocene deposits of Iowa. Bull Geol. Soc. Am., Vol. 20, pp. 133152. 1909.

Cerro Gordo county, peat in.

S. Calvin, in Ia. Geol. Surv., Vol. VII, p. 192. 1896.

Chemical analyses of peat.

Rush Emery, in Geol. Iowa, Vol. Ir, pp. 397-402. 1870.

I. A. Williams, in Iowa Geol. Surv., Vol. XVI, p. 505. 1905.

Chickasaw county, peat in.

S. Calvin, in Ia. Geol. Surv., Vol. XIII, p. 289, 1902.

Davenport, ancient peat bed near.

W. H. Pratt, in Proc. Davenport Acad. Sci., Vol. I, pp. 96-99. 1876.

- C. A. White, in Geol. Iowa, Vol. I, p. 119. 1870.

Emery, Rush.

Chemical analyses of peat. Geol. Iowa, Vol. II, pp. 397-402, 1870.

Forest bed in northeastern Iowa.

W J McGee, in U. S. Geol. Surv., Eleventh Ann. Rept., pp 486-496. 1889-90.

Franklin county, peat in.

I. A. Williams, in Iowa Geol. Surv., Vol. XVI, pp. 495, 504. 1905.

Fulton, A. R.

Peat in Iowa. Iowa: The Home for Immigrants, p. 24. Des Moines, 1870.

Humboldt county, peat in.

T. H. Macbride, in Ia. Geol. Surv., Vol. IX, p. 145. 1898.

Iowa City, ancient peat bed near.

C. A. White, in Geol. Iowa, Vol. I, p. 120. 1870

Keyes, C. R.

Peat, formation of. Ia. Geol. Surv., Vol. II, p. 50. 1894. 
Macbride, T. H.

Peat in Humboldt county. Ia. Geol. Surv., Vol. IX, p. 145. $18^{r} 8$.

Pre-Kansan peat bed. Ia. Acad. Sci., Vol. IV, pp. 63-66. $18 j 6$.

McGee, W J.

Forest bed in northeastern Iowa. U. S. Geol. Surv., Eleve.1th Ann. Rept., pp. 486-496. 1889-90.

Peat, briquetting of.

S. W. Beyer, in Iowa Geol. Surv., Vol. XVIII, p. 28.1907.

Peat, chemical analyses of.

Rush Emery, in Geol. Iowa, Vol. II, pp. 397-402. 1870.

I. A. Williams, in Ia. Geol. Surv., Vol. XVI, p. 505. 1905.

Peat, formation of.

C. R. Keyes, in Ia. Geol. Surv., Vol II, p. 50. 1894,

Peat, funel value of.

S. Calvin, in Iowa Geol. Surv., Vol. XVII, p. 3. 1906.

Peat bed, ancient, in Adair county.

C. A. White, in Geol. Iowa, Vol. I, p. 339. 1870.

$$
\text { , near Davenport. }
$$

W. H. Pratt, in Proc. Davenport Acad. Sci., Vol. I, pp. 96-99. 1876.

C. A. White, in Geol. Iowa, Vol. I, p. 119. 1870.

near Iowa Gity.

C. A. White, in Geol. Iowa, Vol. I, p. 120. 1870.

Peat bed, buried.

T. E. Savage, in Iowa Acad. Sci., Vol. XI, pp. 103-109. 1903.

Peat bed, pre-Kansan.

T. H. Macbride, in Ia. Acad. Sci., Vol. IV, pp. 63-66. 1896.

Peat in Iowa.

A. R. Fulton, in Iowa: The Home For Immigrants, p. 24. Des Moines, 1870.

C. A. White, in Second Ann. Rept. State Geol., pp. 121-135. 1868. in Geol. Iowa, Vol. II, pp. 275-288. 1870.

History of Dallas county, p. 126. Des Moines, 1879. Also in several other county histories.

History of Davis county, p. 130. Des Moines, 1882. Also in several other county histories.

Peat in Pleistocene deposits of Iowa.

S. Calvin, in Bull. Geol. Soc. Am., Vol. 20, pp. 133-152. 1909.

Peat in Buchanan county.

S. Calvin, in Iowa Geol. Surv., Vol. VIII, p. 247. 1897.

Peat in Cerro Gordo county.

S. Calvin, Ibid., Vol. VII, p. 192. 1896.

Peat in Chickasaw county.

S. Calvin, Ibid., Vol. XIII, p. 289. 1902.

Peat in Franklin county.

I. A. Williams, Ibid., Vol. XVI, pp. 495, 504. 1905.

Peat in Humboldt county.

- T. H. Macbride, Ibid., Vol. IX, p. 145. 1898. 
Peat in Worth county.

I. A. Williams, Ibid., Vol. X, p. 375. 1899.

Peat resources of Iowa.

T. E. Savage, Ibid., Bull. 2, pp. 5-21. 1905.

Pratt, W. H.

Peat bed, ancient, near Davenport. Proc. Davenport Acad. Sci., Vol. I, pp. 96-99. 1876

Pre-Kansan peat bed.

T. H. Macbride, in Iowa Acad. Sci., Vol. IV, pp. 63-66. 1896.

Savage, T. E.

Buried peat bed. Ia. Acad. Sci., Vol. XI, pp. 103-109. 1903.

Peat resources of Iowa. Ia. Geol. Surv., Bull. 2, pp. 5-21. 1905.

White, C. A.

Peat bed, ancient, in Adair county. Geol. Iowa, Vol. I, p. 339. 1870.

- near Iowa City. Ibid., p. 120.

— near Davenport. Ibid., p. 119.

Peat in Iowa. Sec. Ann. Rept. State Geol., pp. 121-135. 1868.

Peat in Iowa. Geol. Iowa, Vol. II, pp. 275-288. 1870.

Williams, I. A.

Chemical analysis of peat. Ia. Geol. Surv., Vol. XVI, p. 505. 1905.

Peat in Franklin county. Ibid., Vol. XVI, pp. 495-504. 1905.

Peat in Worth county. Ibid., Vol. X, p. 375. 1899.

Worth county, peat in.

I. A. Williams, in Ia. Geol. Surv., Vol. X, p. 375. 1899. 


$$
\text { - }
$$

